\title{
Investigation On The Optimal Tunable Bi-Stable Clustered Energy Conversion Inspired Dynamic Vibration Absorbers: A Theoretical Study
}

\section{Xingbao Huang}

Shanghai Jiao Tong University

Xiao Zhang

Shanghai Jiao Tong University

Bintang Yang ( $\nabla$ btyang@sjtu.edu.cn )

Shanghai Jiao Tong University https://orcid.org/0000-0001-8394-8558

\section{Research Article}

Keywords: vibration absorption, energy conversion, bi-stable, optimization, impulsive excitation

Posted Date: August 26th, 2021

DOI: https://doi.org/10.21203/rs.3.rs-796801/v1

License: (c) This work is licensed under a Creative Commons Attribution 4.0 International License.

Read Full License 


\title{
Investigation on the optimal tunable bi-stable clustered energy conversion inspired dynamic vibration absorbers: A theoretical study
}

\author{
Xingbao Huang, Xiao Zhang, Bintang Yang* \\ State Key Laboratory of Mechanical Systems and Vibration, School of Mechanical Engineering, Shanghai Jiao \\ Tong University, Shanghai, 200240, China
}

\begin{abstract}
This paper introduces an energy conversion inspired vibration control methodology and presents a representative prototype of tunable bi-stable energy converters. This work is concerned on improving the vibration absorption and energy conversion performance of tunable bi-stable clustered energy conversion inspired dynamic vibration absorbers (EC-DVAs). The deterministic parametric analysis of the energy transfer performance of clustered ECDVAs is conducted. Firstly, nonlinear vibration behaviors including transient energy transfer and snap-through motions are studied, and then effects of EC-DVA number on vibration control is investigated. Furthermore, the optimal computation based on adjusting the length ratio (namely bi-stable potential barrier height) is developed to obtain the maximum energy conversion efficiency of clustered EC-DVAs and the minimum residual kinetic energy of the primary system considering different number of clustered EC-DVAs. Moreover, the optimal calculation based on optimal EC-DVA number is also developed to achieve the most excellent vibration absorption and energy conversion performance. Finally, the optimal calculation based on optimal mass ratio is conducted. Numerical simulations show that when the total mass ratio is constant the snap-through motions of each EC-DVA depend remarkably on EC-DVA number; the energy conversion efficiency and residual kinetic energy after dynamic length ratio optimization is independent on ambient input energy and EC-DVA number; The energy conversion efficiency and vibration absorption performance based on optimal EC-DVA number maintain high efficiency and stable when the ambient input energy or the potential energy of clustered EC-DVAs varies. The optimal mass ratio is large when the system's potential barrier is too large and the ambient input energy is small. Therefore, the presented tunable bistable system of clustered EC-DVAs with appropriate bi-stable potential function and proposed optimization strategies is a potential alternative for vibration control of mechanical components exposed to varying impulses.
\end{abstract}

Keywords: vibration absorption; energy conversion; bi-stable; optimization; impulsive excitation

\section{Highlights:}

1. A tunable bi-stable clustered energy conversion inspired dynamic vibration absorber (EC-DVAs) is proposed.

2. The transient energy transfer performance and nonlinear vibration behaviors of EC-DVAs are discussed.

3. The optimization strategy based on modulating the bi-stable potential energy is developed to obtain the optimal EC-DVAs.

4. The optimization strategy based on adjusting number of clustered EC-DVAs is conducted to obtain the optimal EC-DVAs.

5. The optimization strategy based on optimal mass ratio is implemented to obtain the optimal EC-DVAs.

\section{Introduction}

Catastrophic vibrations caused by resonances extensively exist in multifarious engineering applications. In order to inhibit and easily control the unwanted vibrations, passive, semi-active and active vibration control strategies are developed to dispose this issue. Generally, the semi-active or active control technique needs advanced adaptive algorithms and artificial input of considerable energy for vibration mitigation. Combining the merits of passive and 
semi-active vibration control strategies, parameter adjustable passive vibration absorbers are the desired alternatives for vibration suppression and energy transfer. Dynamic vibration absorbers(DVAs) have been widely employed to sorts of civil or mechanical structures in order to mitigate vibration yielded by seismic excitations [1,2,3], flow induced vibrations (FIV) [4,5,6], unbalanced rotating machinery [7,8], dynamic vibration caused from vehicle traffic $[9,10,11]$, vibrations caused by thermal alternating loads $[12,13]$, and other causes. A classic DVA is always composed of a single degree-of-freedom (SDOF) mass-spring-damper system, which is only effective when the linear primary system is excited by stable single-frequency excitations. Over the past few decades, the vibration absorption performance and robustness of the classic DVA have been improved by introducing multiple DVAs that are arranged in parallel or in series. Kim and Lee developed a general design guide for optimal linear multiple DVAs including constraints on the frequency ratio, damping ratio, and mass distribution in order to cover practical concerns such as space limitations, transportation, and ease of handling[14]. The DVAs arranged in series are more effective and robust than their counterparts arranged in parallel when the mass ratio for them is unaltered, moreover, the DVA series can obtain much broader effective operating bandwidth of the protected system compared with other DVAs [15]. However, the vibration suppression performance of these mechanical devices is limited by the timeworn mass ratio as well as the correct tuning of their two vibration modes. Therefore, adding new mechanical elements or electromagnetic devices to passive DVAs that function as energy conversion components is a potential approach to implementing wideband vibration absorption.

Multi-stable DVAs are novel nonlinear DVAs that perform excellently in vibration absorption of a primary system excited by transient impulsive excitations. Due to the presence of potential well with different depths, the transient input energy can be easily entrapped in one of these wells, and the energy conversion efficiency is significantly enhanced by means of intensive snap-through motions (mixture of cross-well and in-well motions). Chiacchiari et al.[16,17] investigated the potential benefit of the bi-stable element coupled to a directly excited primary system for conversion broadband and low-amplitude vibration energy such as single and repeated impulses, and explored three main dynamic regimes, namely periodic cross-well, aperiodic (chaotic) cross-well, and in-well oscillations. Huang and Yang [18] studied the energy conversion performances and energy trapping capacity of a tristable DVA under impulsive excitations and harmonic vibrations in comparison with their counterparts of a bi-stable DVA. Nguyen et al.[19] developed detailed models for both mono-stable and bi-stable magnetic spring based vibration energy harvester configurations, where the bi-stability is caused by introducing a cluster of peripheral solid magnets. In fact, the ambient excitations is time-varying, unpredictable and aperiodic, thereby, how to obtain the optimum vibration suppression performance and the uppermost energy conversion efficiency is an optimization problem including clear understandings of structure and physical parameters of a DVA. Some researchers have made considerable efforts to optimize vibration control[20,21,22]. Hua et al.[20] proposed a beam-based DVA and optimized it for minimizing the resonant vibration of a general structure by means of fixed-points theory. Shen et al.[21] presented a novel DVA with grounded stiffness element and amplifying mechanism, and obtained optimum frequency ratio, optimum grounded stiffness ratio and optimum damping ratio of the DVA based on the fixed-point theory. He et al.[22] studied vibration reduction properties of the periodically stiffened panels by using the datadriven optimization method which is used to investigate the multi-objective structural optimization problem for minimizing the weight and mean square velocity simultaneously. However, most reported optimization methods are focused on the parameters of a linear DVA, and very few attentions are paid on the optimization strategy of multistable DVAs that are fond candidates for nonlinear vibration suppression.

The motivation of this paper is to develop an optimization methodology for energy reuse and vibration suppression. In this work a tunable bi-stable system composed of clustered vibration-based energy harvesters and dynamic vibration absorbers (EC-DVAs) is proposed. The potential function of the proposed bi-stable system can be readily designed and modulated by means of adjusting the constringent format of negative stiffness spring 
configuration. Two optimization strategies in terms of maximizing the energy conversion efficiency of EC-DVA arrays and minimizing the residual kinetic energy ratio of the protected system are developed. Firstly, the influencing mechanism of the number of clustered EC-DVAs is investigated with the application of a single impulsive excitation scenario. Furthermore, the optimal computation based on adjusting the length ratio is developed to obtain the maximum energy conversion efficiency of clustered EC-DVAs and the minimum residual kinetic energy of the primary system considering different number of clustered EC-DVAs. Moreover, the optimal calculation based on optimal number of clustered EC-DVAs is also implemented to achieve the most excellent vibration absorption and energy conversion performance of tunable clustered EC-DVAs. Finally, the optimization strategy based on optimal mass ratio is conducted to obtain the optimum vibration absorption and energy conversion performances.

\section{Mathematical description}

In this work the clustered EC-DVAs coupled to a grounded and weakly damped linear primary system are considered. Figure 1 gives schematic configuration of the clustered EC-DVAs that contain uniform or different ECDVA cells. From Figure 1 it can be easily found that the coupling stiffness between each EC-DVA cell and the primary system can be governed by modulating the potential energy of the clustered EC-DVAs. Due to the advanced energy trapping performances of bi-stable systems, bi-stable structures become the favored candidates for nonlinear DVAs. For a conventional nonlinear DVA, the retained energy in DVA is mainly dissipated by damping elements, that is to say, the vibration suppression efficiency of the primary system remarkably depends on the utilization of damping elements. However, bi-stable energy harvesters have excellent energy trapping and conversion performances. Thereby, the proposed EC-DVA is a novel bi-stable system which is composed of an electromagnetic energy harvester and a DVA mass. Figure 2 describes schematic description of a tunable bi-stable EC-DVA, from which it can be easily seen that a bi-stable system can be obtained and tuned through controlling the motion of two sliders. Figure 3 presents the tunable potential energy of EC-DVA with different length ratio $\sigma$, from which it can be seen that the height of potential barrier increases when length ratio $\sigma$ decreases. When the length ratio $\sigma$ is set to 1 , the bi-stable potential energy of EC-DVA is degraded into monostability, as shown in Figure 3. It is well acknowledged that the trigger threshold of a bi-stable system is seriously dependent on the potential barrier height which determines the minimum needed ambient input energy. Therefore, an appropriate bi-stable system can be obtained by designing a tunable potential energy of the concerned system.

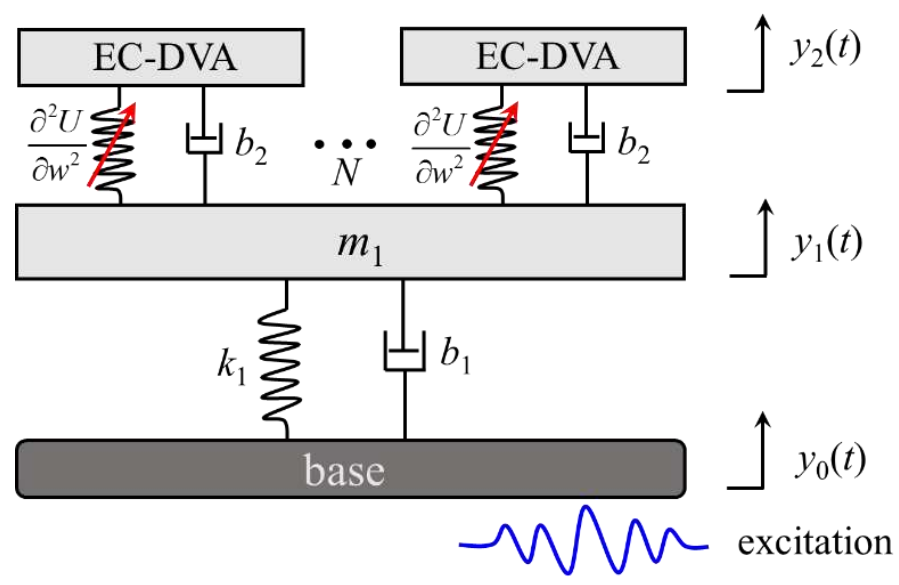

Figure 1 Schematic configuration of the clustered EC-DVAs 

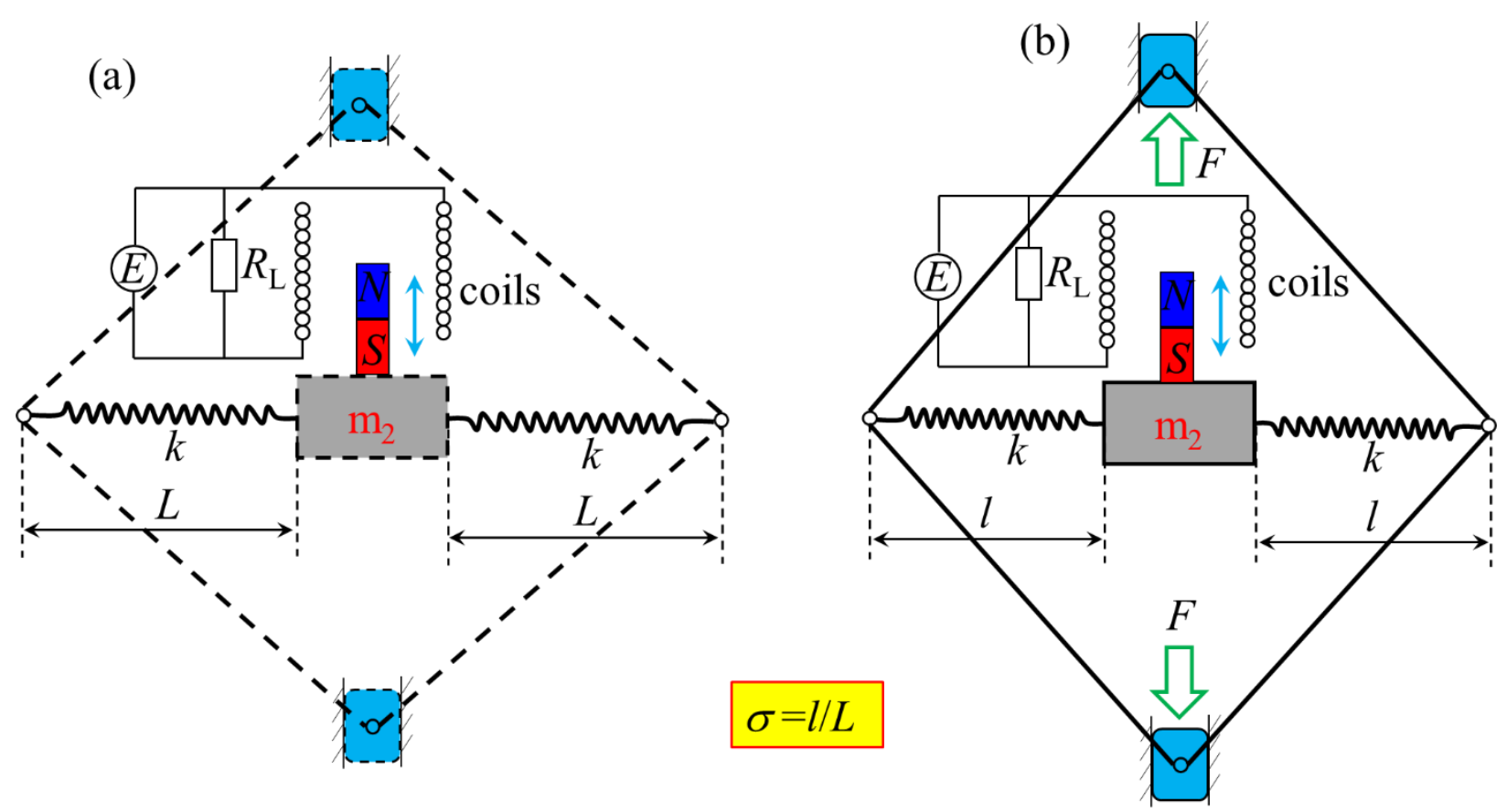

Figure 2 Schematic description of a tunable bi-stable EC-DVA

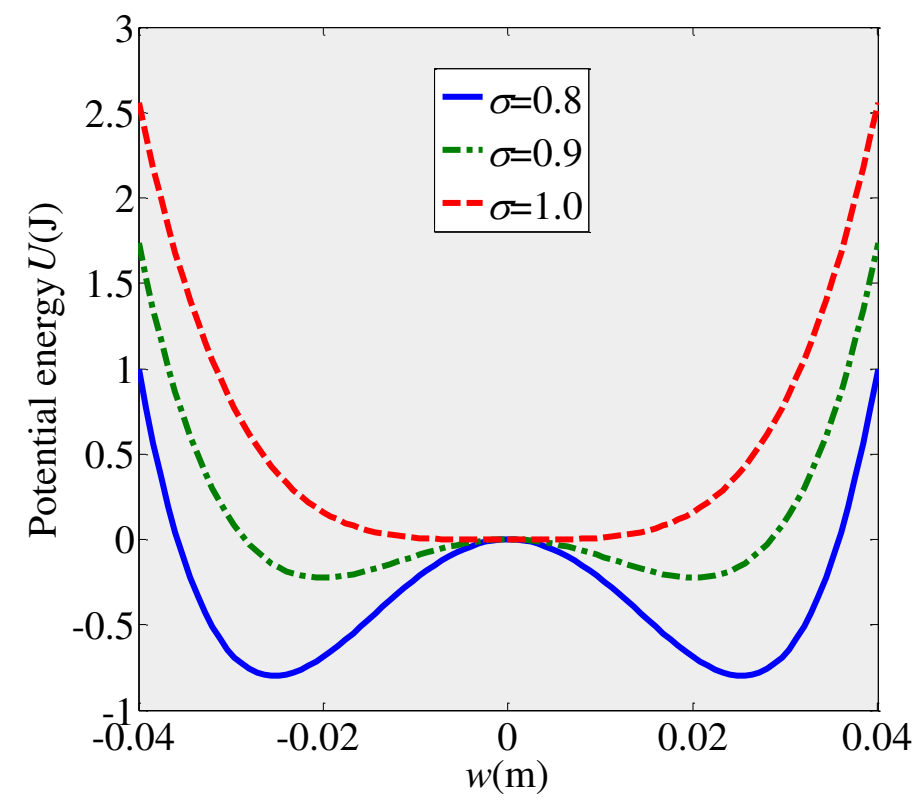

Figure 3 The tunable potential energy of EC-DVA

\section{Governing equations}

In this section a coupled dynamic system composed of a linear primary system, clustered EC-DVAs and multiple electromagnetic generators is considered. In order to obtain the maximum energy conversion efficiency and the minimum residual kinetic energy, optimization algorithm of the optimal number of EC-DVAs and length ratio $\sigma$ is developed. The system of coupled equations can be expressed by 


$$
\left\{\begin{array}{l}
m_{1} \ddot{y}_{1}+b_{1} \dot{y}_{1}+k_{1} y_{1}-\sum_{i=1}^{N}\left[\left(b_{2}^{(i)}+b_{e}^{(i)}\right) \dot{w}_{i}+\frac{\partial U_{i}}{\partial w_{i}}\right]=F(t) \\
m_{2}^{(i)}\left(\ddot{w}_{i}+\ddot{y}_{1}\right)+\left(b_{2}^{(i)}+b_{e}^{(i)}\right) \dot{w}_{i}+\frac{\partial U_{i}}{\partial w_{i}}=0 \\
\dot{E}_{i}(Q, T)-\psi_{i} \dot{w}_{i}=0
\end{array}\right.
$$

where $(\cdot$.$) and (\cdot)$ denote the second-order differential and first-order differential, respectively. $m_{1}$ and $m_{2}^{(i)}$ denote the primary structure mass and the $i^{\text {th }}$ EC-DVA cell mass, respectively. $b_{1}$ and $b_{2}^{(i)}$ denote the primary structure damping coefficient and the $i^{\text {th }}$ EC-DVA cell damping coefficient, respectively. $b_{e}^{(i)}$ and $k_{e}^{(i)}$ are the electromechanical damping coefficient and transduction factor of the $i^{\text {th }}$ EC-DVA cell, respectively. $y_{1}$ is the absolute displacement of primary structure mass; $w_{i}=y_{2}^{(i)}-y_{1}$ is the relative displacement of the $i^{\text {th }}$ EC-DVA cell mass. $U_{i}$ is the potential energy of the $i^{\text {th }}$ EC-DVA cell. $\partial U_{i} / \partial w_{i}$ is the conservative force of the $i^{\text {th }}$ EC-DVA cell. Hereby, $\dot{E}_{i}(Q, T)$ denotes the converted energy (including electric energy, heat energy, and etc.), for electric energy the form of $\dot{E}_{i}(Q, T)$ is $\dot{Q}_{i}$. The conversion coefficient $\psi_{i}$ in electromagnetic conversion mechanism stands for $b_{e}^{(i)} / k_{e}^{(i)} . F(t)$ is the ambient excitation force, herein denotes transient impulsive force. For the proposed tunable bi-stable system, whose conservative force can be calculated by

$$
\frac{\partial U_{i}}{\partial w_{i}}=2 k w_{i}\left(1-\frac{L}{\sqrt{w_{i}^{2}+l_{i}^{2}}}\right)
$$

For the sake of facilitating the efficient numerical computation, all parameters are nondimensionalized and the dimensionless parameters of the system are defined in the following forms:

$$
\tau=t / C_{t}, w_{i}=L u_{i}, y_{1}=L x, \sigma_{i}=l_{i} / L, Q_{i}=C_{q}^{(i)} q_{i}, C_{t}=\sqrt{m_{1} / k_{1}}, C_{q}^{(i)}=\frac{m_{2}^{(i)} L}{k_{e}^{(i)}} \sqrt{\frac{k_{1}}{m_{1}}}, a_{i}=\sqrt{-\xi_{i} / \alpha_{i}}, u_{i}=z_{i}+a_{i}
$$

By introducing the dimensionless time $\tau=t / C_{t}$, the system (1) can be rewritten as

$$
\left\{\begin{array}{l}
x^{\prime \prime}+\lambda x^{\prime}+x-\sum_{i=1}^{N} \mu_{i}\left[\left(\beta_{i}+\zeta_{i}\right) z_{i}^{\prime}-2 \xi_{i} z_{i}+3 a_{i} \alpha_{i} z_{i}^{2}+\alpha_{i} z_{i}^{3}\right]=\gamma_{i} f(\tau) \\
z_{i}^{\prime \prime}+x^{\prime \prime}+\left(\beta_{i}+\zeta_{i}\right) z_{i}^{\prime}-2 \xi_{i} z_{i}+3 a_{i} \alpha_{i} z_{i}^{2}+\alpha_{i} z_{i}^{3}=0 \\
q_{i}^{\prime}-\beta_{i} z_{i}^{\prime}=0
\end{array}\right.
$$

where 


$$
\begin{aligned}
& \lambda=\frac{b_{1}}{m_{1}} \sqrt{\frac{m_{1}}{k_{1}}}, \mu_{i}=\frac{m_{2}^{(i)}}{m_{1}}, \beta_{i}=\frac{b_{e}^{(i)}}{m_{2}^{(i)}} \sqrt{\frac{m_{1}}{k_{1}}}, \zeta_{i}=\frac{b_{2}^{(i)}}{m_{2}^{(i)}} \sqrt{\frac{m_{1}}{k_{1}}}, \\
& \xi_{i}=\frac{2 k m_{1}}{m_{2}^{(i)} k_{1}}\left(1-\frac{1}{\sigma_{i}}\right), \alpha_{i}=\frac{k m_{1}}{m_{2}^{(i)} k_{1}} \frac{1}{\sigma_{i}^{3}}, \gamma_{i}=\frac{m_{1}}{k_{1} L m_{2}^{(i)}}
\end{aligned}
$$

A single impulsive excitation scenario is studied in this work. The multiple system (3) is initially at rest at $\tau=0_{-}$, where the $0_{-}$denotes the moment just prior to instantaneous zero. A single impulse $\gamma f(\tau)=V_{0} \delta(\tau)$ is then applied to the linear primary structure at $\tau=0$, where $\delta(\tau)$ is the Dirac delta function. Hereby, the $V_{0}=v_{0} \cdot C_{t} / L$ is the dimensionless impulsive velocity defined in the normalized system. With permission of an instantaneously applied force assumption, the equations of motion (3) are complemented by the following initial conditions:

$$
x\left(0^{+}\right)=0, \quad \dot{x}\left(0^{+}\right)=V_{0}, \quad z\left(0^{+}\right)=0, \quad \dot{z}\left(0^{+}\right)=-V_{0}, \quad q\left(0^{+}\right)=0
$$

Electric power expressions can be employed to describe the transient energy conversion performance of the energy conversion system. The instantaneous power extracted by the transduction mechanism, such as dissipated across the resistive element in the coupled circuit, can be calculated by

$$
P\left(t, v_{0}\right)=\sum_{i=1}^{N} R_{L} \dot{Q}_{i}^{2}=\sum_{i=1}^{N}\left(\frac{\left(k_{e}^{(i)}\right)^{2}-R_{c} b_{e}^{(i)}}{\left(k_{e}^{(i)}\right)^{2}} \cdot k_{1} L^{2} \cdot \sqrt{\frac{k_{1}}{m_{1}}}\right) \frac{\mu_{i}}{\beta_{i}}\left(q_{i}^{\prime}\right)^{2}
$$

where the term in brackets has units of Watts, whereas the term $\mu_{i} / \beta_{i} q_{i}^{2}$ is the non-dimensional power. Then the total energy harvested following a single impulsive excitation scenario can be correspondingly obtained by

$$
E_{h}\left(t, v_{0}\right)=\int_{0}^{t} P\left(t^{\prime}, v_{0}\right) \mathrm{d} t^{\prime}=\int \sum_{i=1}^{N} R_{L} \dot{Q}_{i}^{2}=\int \sum_{i=1}^{N}\left(\frac{\left(k_{e}^{(i)}\right)^{2}-R_{c} b_{e}^{(i)}}{\left(k_{e}^{(i)}\right)^{2}} \cdot k_{1} L^{2} \cdot \sqrt{\frac{k_{1}}{m_{1}}}\right) \frac{\mu_{i}}{\beta_{i}}\left(q_{i}^{\prime}\right)^{2}
$$

The total input energy after a single impulsive excitation hereby is defined as the initial kinetic energy of the primary system that is exchanged by the ambient impact. Therefore, the energy conversion efficiency $\eta_{\mathrm{h}}$ can be expressed by

$$
\eta_{h}\left(t, v_{0}\right)=\frac{E_{h}\left(t, v_{0}\right)}{\frac{1}{2} m_{1} v_{0}^{2}} \times 100 \%
$$

The residual kinetic energy ratio of the primary system after a period time $t_{f}$ with respect to the input energy can be calculated by

$$
\eta_{k}\left(t_{f}, v_{0}\right)=\frac{T_{P S}\left(t_{f}, v_{0}\right)}{\frac{1}{2} m_{1} v_{0}^{2}} \times 100 \%=\frac{\frac{1}{2} m_{1} v_{1}^{2}}{\frac{1}{2} m_{1} v_{0}^{2}} \times 100 \%=\frac{v_{1}^{2}}{v_{0}^{2}} \times 100 \%
$$

The transient kinetic energy of EC-DVAs, transient potential energy of EC-DVAs, transient kinetic energy of 
the primary system and transient potential energy of the primary system can be expressed by

$$
\begin{gathered}
T_{P S}\left(t, v_{0}\right)=\frac{1}{2} m_{1} v_{1}^{2} \\
U_{P S}\left(t, v_{0}\right)=\frac{1}{2} k_{1} y_{1}^{2} \\
T_{E C-D V A}\left(t, v_{0}\right)=\frac{1}{2} m_{2}^{(i)}\left(v_{2}^{(i)}\right)^{2} \\
U_{E C-D V A}\left(t, v_{0}\right)=\int 2 k w_{i}\left(1-\frac{L}{\sqrt{w_{i}^{2}+l_{i}^{2}}}\right) \mathrm{d} w_{i}
\end{gathered}
$$

Due to the clustered EC-DVAs are a series of nonlinear oscillators, the EC-DVA is actually a nonlinear energy sink (NES). Therefore, the entrapped energy ratio of clustered EC-DVAs can be calculated by

$$
\eta_{N E S}=\frac{\sum T_{E C-D V A}\left(t, v_{0}\right)+\sum U_{E C-D V A}\left(t, v_{0}\right)}{T_{P S}\left(t, v_{0}\right)+U_{P S}\left(t, v_{0}\right)+\sum T_{E C-D V A}\left(t, v_{0}\right)+\sum U_{E C-D V A}\left(t, v_{0}\right)} \times 100 \%
$$

Accordingly, the transient residual kinetic energy ratio in primary system can be computed by

$$
\eta_{r}=\frac{T_{P S}\left(t, v_{0}\right)}{T_{P S}\left(t, v_{0}\right)+U_{P S}\left(t, v_{0}\right)+\sum T_{E C-D V A}\left(t, v_{0}\right)+\sum U_{E C-D V A}\left(t, v_{0}\right)} \times 100 \%
$$

\section{Numerical methodology}

In this paper a package of optimization algorithms are developed to obtain the maximum energy conversion efficiency and minimum residual kinetic energy. The fourth-order Runge-Kutta method is employed to solve the system of coupled equations. The optimization algorithms hereby are divided into two parts: calculating the optimal length ratio and calculating the optimal number of EC-DVAs. Figure 4 and Figure 5 presents the optimization algorithm flow of obtaining the maximum energy conversion efficiency and the minimum residual kinetic energy by means of modulating the optimal parameter 2 which denote the mass ratio, length ratio or number of EC-DVAs, respectively. Before the input energy evolution one of the concerned parameters (mass ratio, length ratio and number of EC-DVAs) is fixed. From Figure 4 and Figure 5 it can be seen that outermost loop and middle loop are functioned as parameterized scanning approaches. The innermost loop is employed to obtain the maximum energy conversion efficiency and the minimum residual kinetic energy, meanwhile, label the optimal parameter 2 to serve as active regulation criterion when the ambient input energy varies. 


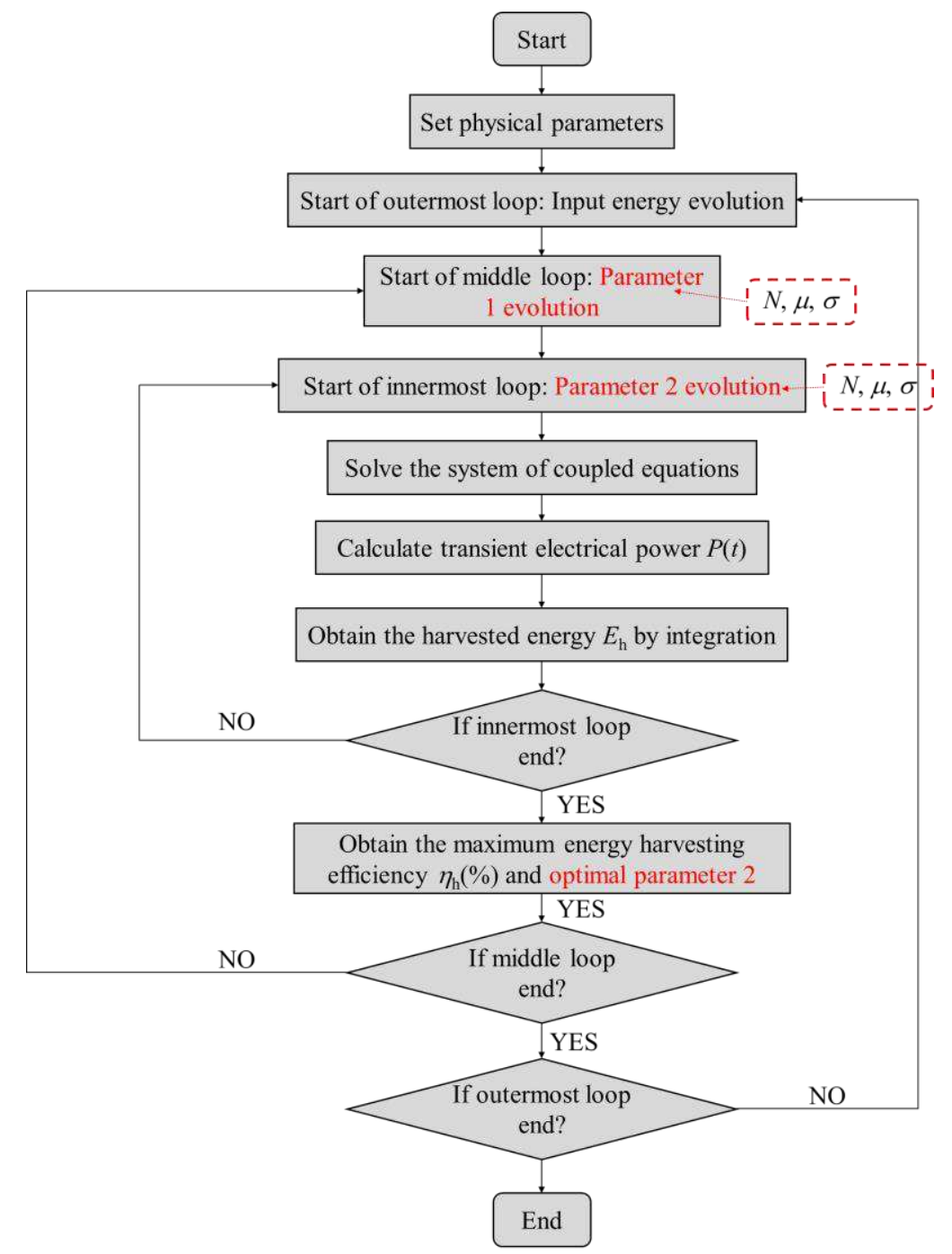

Figure 4 The optimization algorithm flow of obtaining the maximum energy conversion efficiency 


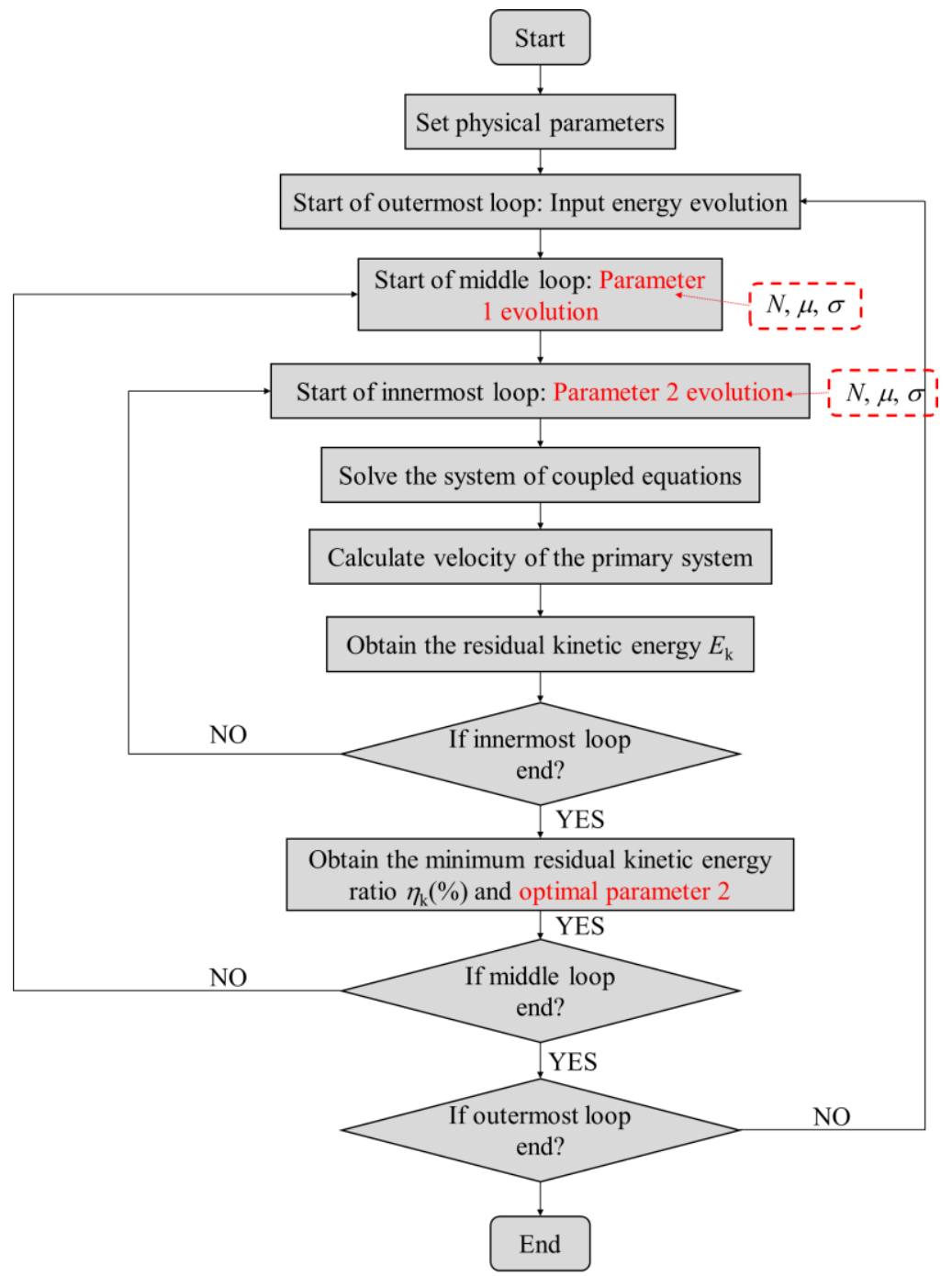

Figure 5 The optimization algorithm flow of obtaining the minimum residual kinetic energy

\section{Results and discussion}

In this section we firstly study the physical behaviors of clustered tunable bi-stable EC-DVAs by means of analyzing the transient targeted energy transfer (TET) performance, energy conversion efficiency, residual kinetic energy ratio and snap-through motion behaviors. Furthermore, the influence of number of EC-DVAs on the energy conversion efficiency and vibration absorption efficiency of the clustered EC-DVAs is investigated. The determination of total mass ratio is based on the compromise consideration of clustered EC-DVAs mass constraint and the effectiveness of bi-stable system mass ratio, thereby the total mass ratio $\mu N$ is set to 0.2 . Table 1 presents parameters of the clustered EC-DVAs system. In order to well understand the effects of number of EC-DVAs on the impulse response of primary system and motion evolution of each EC-DVA cell, the wavelet transform spectra and phase portrait are studied and discussed. Additionally, optimization strategies in terms of vibration absorption and energy conversion performance of clustered EC-DVAs are proposed and analyzed. The optimization objective is chosen as the maximum energy conversion efficiency or the minimum residual kinetic energy ratio, here the optimized parameter is mass ratio, length ratio or number of EC-DVAs.

Table 1 Parameters of the clustered EC-DVAs system

\begin{tabular}{lr}
\hline Description, symbol & Value \\
\hline Mass of primary structure, $m_{1}$ & $7.1 \mathrm{~kg}$ \\
\hline Linear viscous damping of primary structure, $b_{1}$ & $0.38 \mathrm{~N} \cdot \mathrm{s} / \mathrm{m}$ \\
\hline \multicolumn{2}{c}{9}
\end{tabular}




\begin{tabular}{lr}
\hline Linear stiffness of primary structure, $k_{1}$ & $19889.25 \mathrm{~N} / \mathrm{m}$ \\
\hline Linear viscous damping in the coupling, $b_{2}$ & $0.31 \mathrm{~N} \cdot \mathrm{s} / \mathrm{m}$ \\
\hline Coil resistance, $R_{\mathrm{c}}$ & $32.1 \Omega$ \\
\hline Load resistance, $R_{\mathrm{L}}$ & $50 \Omega$ \\
\hline Transduction factor, $k_{\mathrm{e}}$ & $15 \mathrm{~T} \cdot \mathrm{m}$ \\
\hline The original length of equivalent spring, $L$ & $0.05 \mathrm{~m}$ \\
\hline The base stiffness of equivalent spring, $k$ & $500 \mathrm{~N} / \mathrm{m}$ \\
\hline Electromechanical damping coefficient, $b_{\mathrm{e}}$ & $2.74 \mathrm{~N} \cdot \mathrm{s} / \mathrm{m}$ \\
\hline The synthesis mass ratio of the system, $\mu N$ & 0.2 \\
\hline
\end{tabular}

5.1 The energy capture performance and nonlinear dynamics of the clustered tunable bi-stable EC-DVAs

In this section we study the energy conversion and vibration absorption performance of clustered tunable bistable EC-DVAs, and investigate the intra-well and inter-well motion behaviors under different conditions such as different input energy, different length ratio and different number of EC-DVA cells. Hereby, the default number of EC-DVA cells is 4, and the default synthesis mass ratio is set to 0.2. Figure 6 and Figure 7 present the energy harvesting efficiency and residual kinetic energy ratio measured at dimensionless time $\tau=60$ under different input energy, respectively. From Figure 6 and Figure 7 it can be easily found that efficient energy conversion can be achieved when $\sigma$ ranges approximately from 0.6 to 0.8 , and correspondingly the residual kinetic energy in the primary system is significantly decreased when $\sigma$ ranges approximately from 0.6 to 0.8 . Additionally, it can be also seen in Figure 6 and Figure 7 that the optimum energy conversion (saturated state) and vibration absorption performance are synchronously obtained when the impact velocity is $2.6464 \mathrm{~m} / \mathrm{s}$.

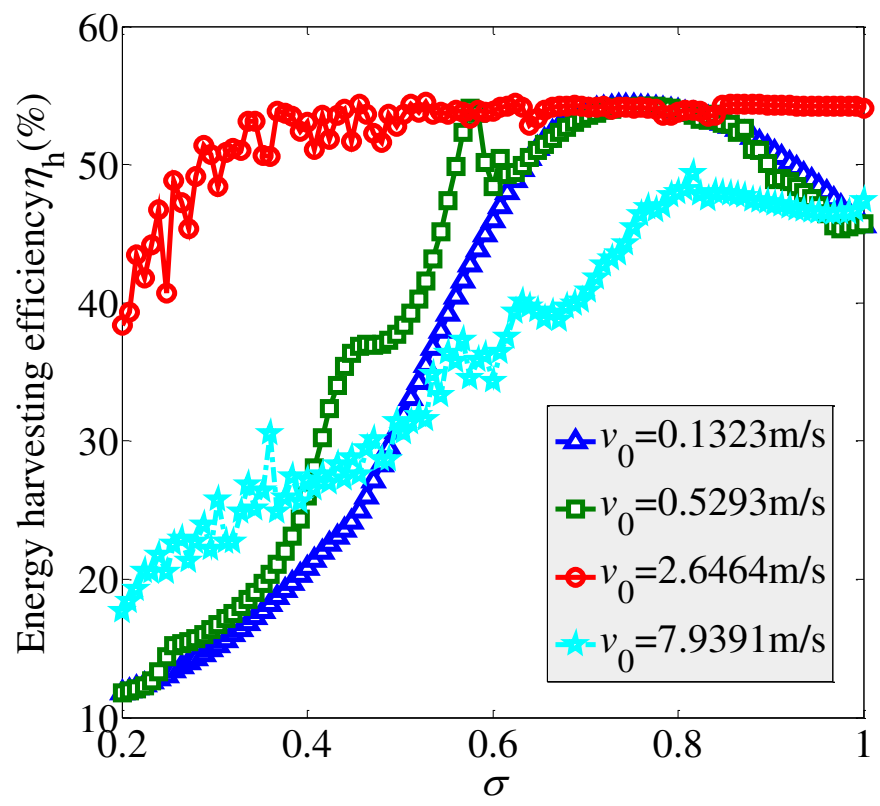

Figure 6 The energy harvesting efficiency under different input energy 


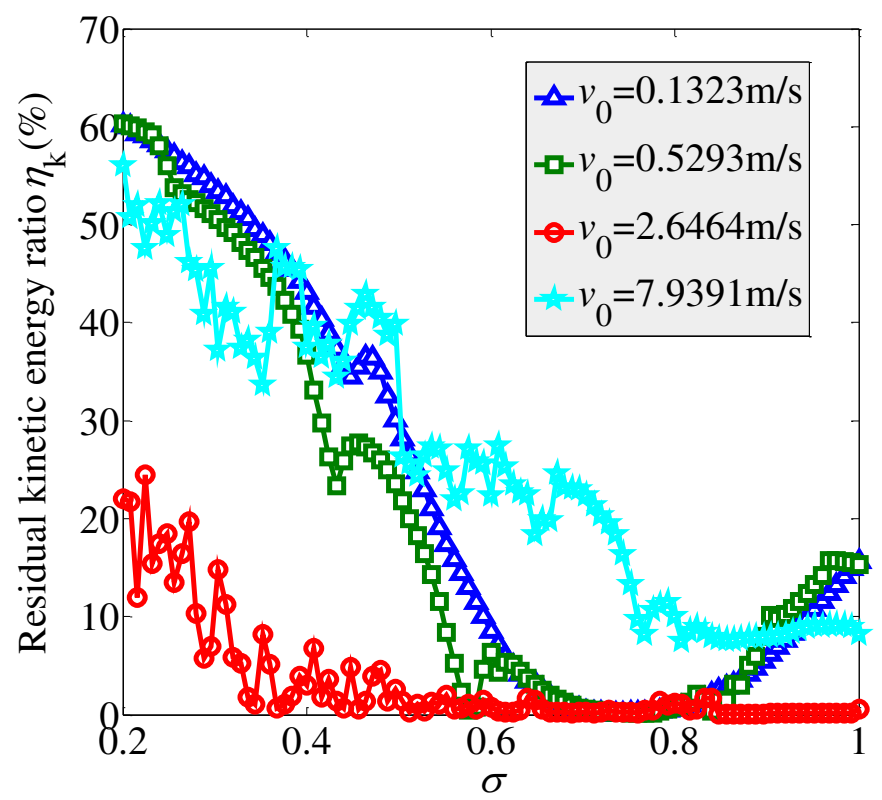

Figure 7 The residual kinetic energy ratio under different input energy

Figure 8 shows the transient entrapped energy ratio in the NES system under different input energy from small level to large level. From Figure 8 it can be easily seen that the transient TET performance is unsatisfactory when the input energy is too small or too large, and it can be also found that the cubic nonlinearity only works when the input energy is large. Additionally, it can be observed in Figure 8 that the TET performance is heavily dependent on the length ratio. Therefore, it is necessary to investigate the relationship between optimal length ratio and optimum energy conversion under different input energy.

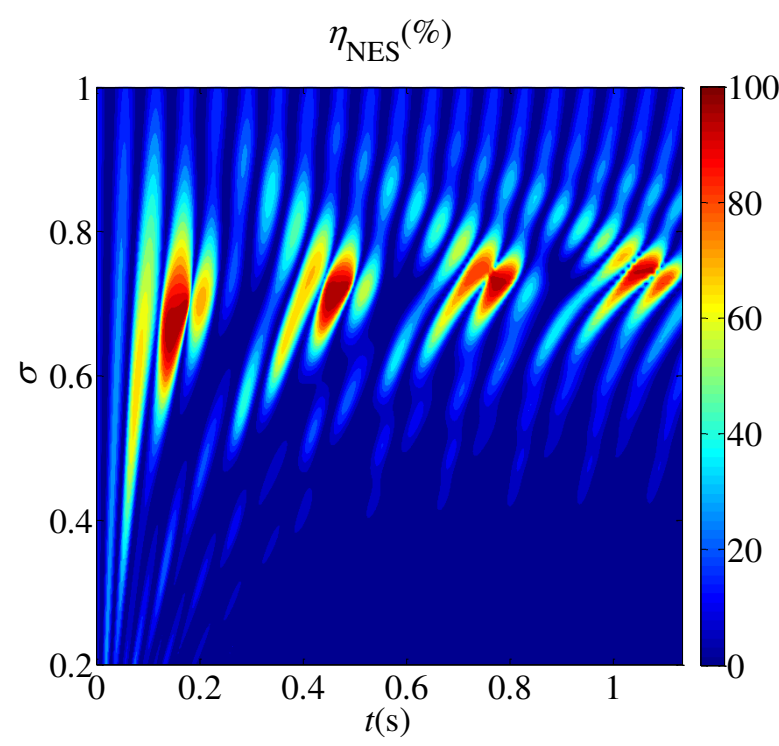

(a)

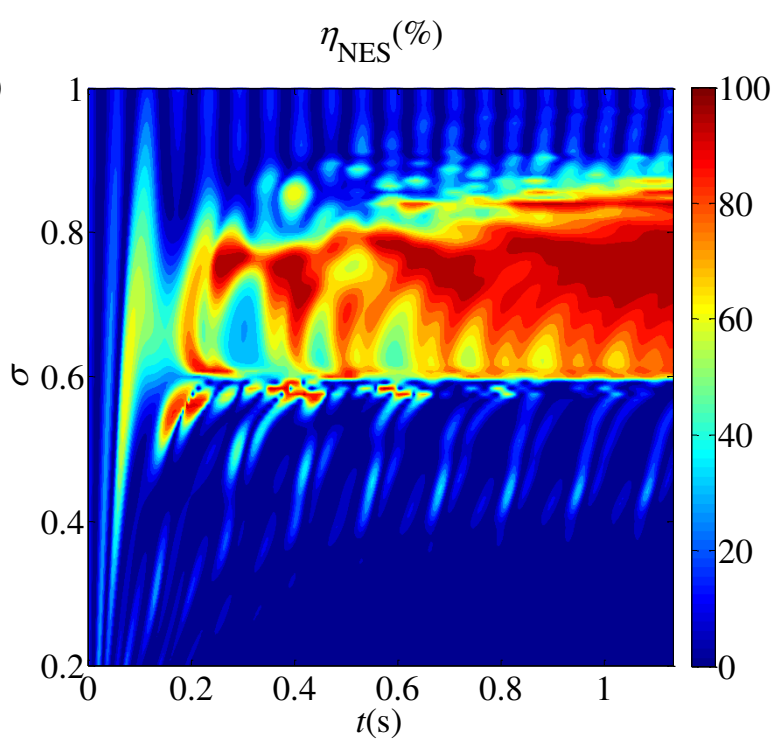

(b) 


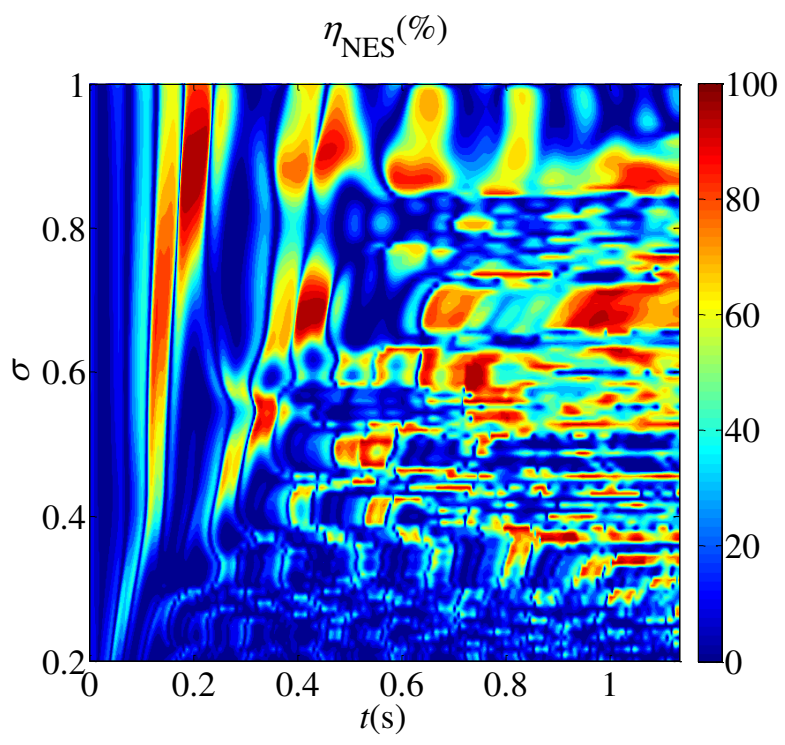

(c)

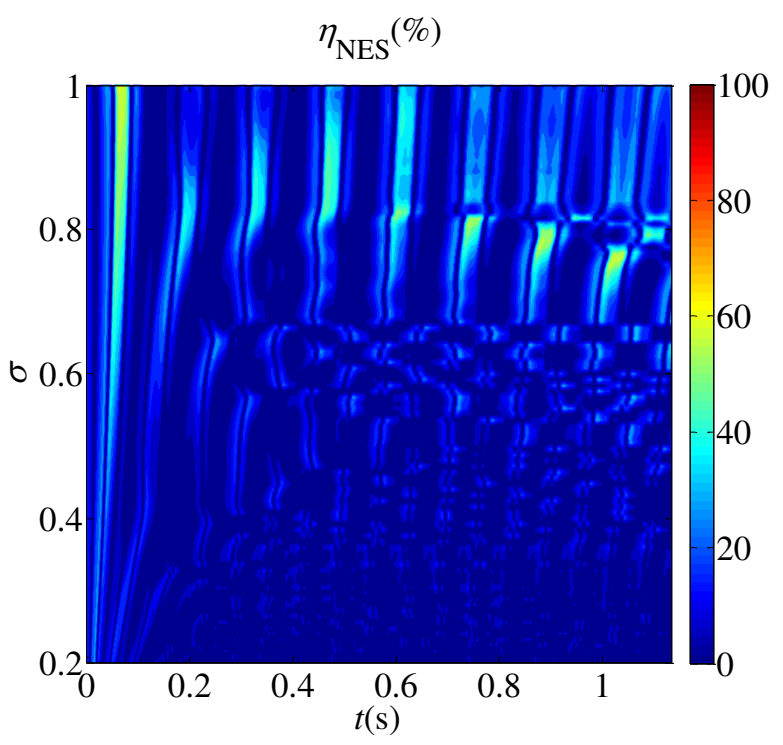

(d)

Figure 8 The transient entrapped energy ratio in the NES system under different input energy. (a) $v_{0}=0.1323$; (b) $v_{0}=0.5293 ;$ (c) $v_{0}=2.6464 ;$ (d) $v_{0}=7.9391$.

In order to understand the intra-well and inter-well motions under different structural configurations, the displacement time histories of the primary system and NES system with different length ratio or different number of EC-DVA cells are studied. Figure 9 gives the nonlinear motion behaviors of the primary system and NES system with a deterministic impulsive excitation $v_{0}=1.3232 \mathrm{~m} / \mathrm{s}$. From Figure 9 it can be seen that compared with cubic nonlinearity the bi-stable mechanism can improve the TET performance by means of triggering snap-through motions between the two stable equilibrium positions, moreover, the vibration suppression performance of the primary system with $\sigma=0.75$ is much better than its counterpart with $\sigma=0.5$. The reason for satisfactory vibration suppression performance with $\sigma=0.75$ is due to the critical energy threshold of $\sigma=0.75$ is lower than the case of $\sigma=0.5$, which can be easily understood in Figure 9(b). For the sake of exploring the influence of number of EC-DVA cells on the vibration suppression performance, the nonlinear motion behaviors of the primary system and NES system with different number of EC-DVA cells are evaluated, as shown in Figure 10 and Figure 11. Herein, the length ratio is set to 0.8 . From Figure 10 and 11 it can be seen that the vibration suppression performance of the primary system depends greatly on number of EC-DVA cells, and the TET performance is remarkable when eight uniform EC-DVA cells are applied to the system. Additionally, the nonlinear oscillators rest at one stable equilibrium position when the impulsive excitation is too small to reach the critical threshold to trigger the transition from intra-well to high-energy inter-well mechanism, as shown in Figure 10(b). It can be evidently found that high-energy inter-well motions can be achieved by increasing the input energy, as described in Figure 11(b).

With the understandings of the effects of length ratio and EC-DVA number on the energy conversion and vibration absorption performance under various impulsive excitations, it can be very unambiguous that the vibration control performance can be improved and optimized through modulating structural configuration matching the optimal length ratio and EC-DVA number. Therefore, optimization algorithms whose objective function is energy conversion maximization and residual kinetic energy minimization are of importance for adaptive vibration control under time-varying environment. 


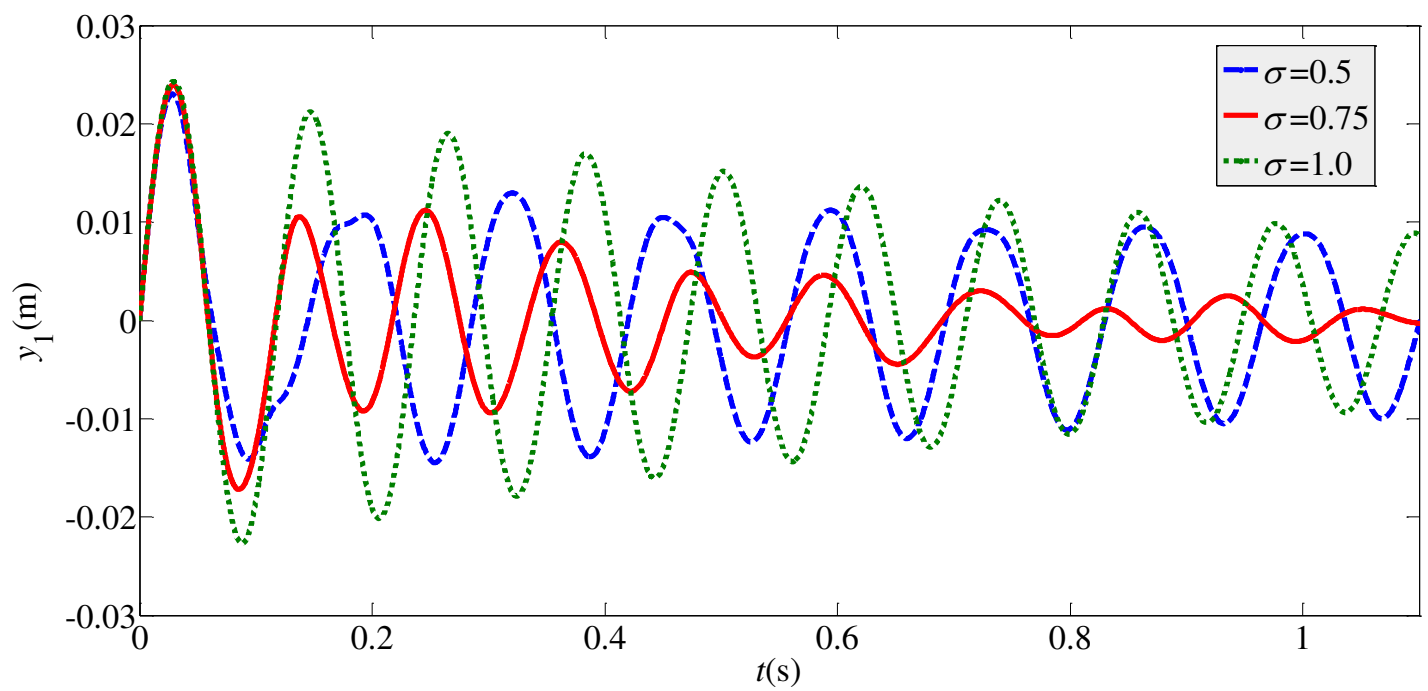

(a) The displacement time history of the primary system

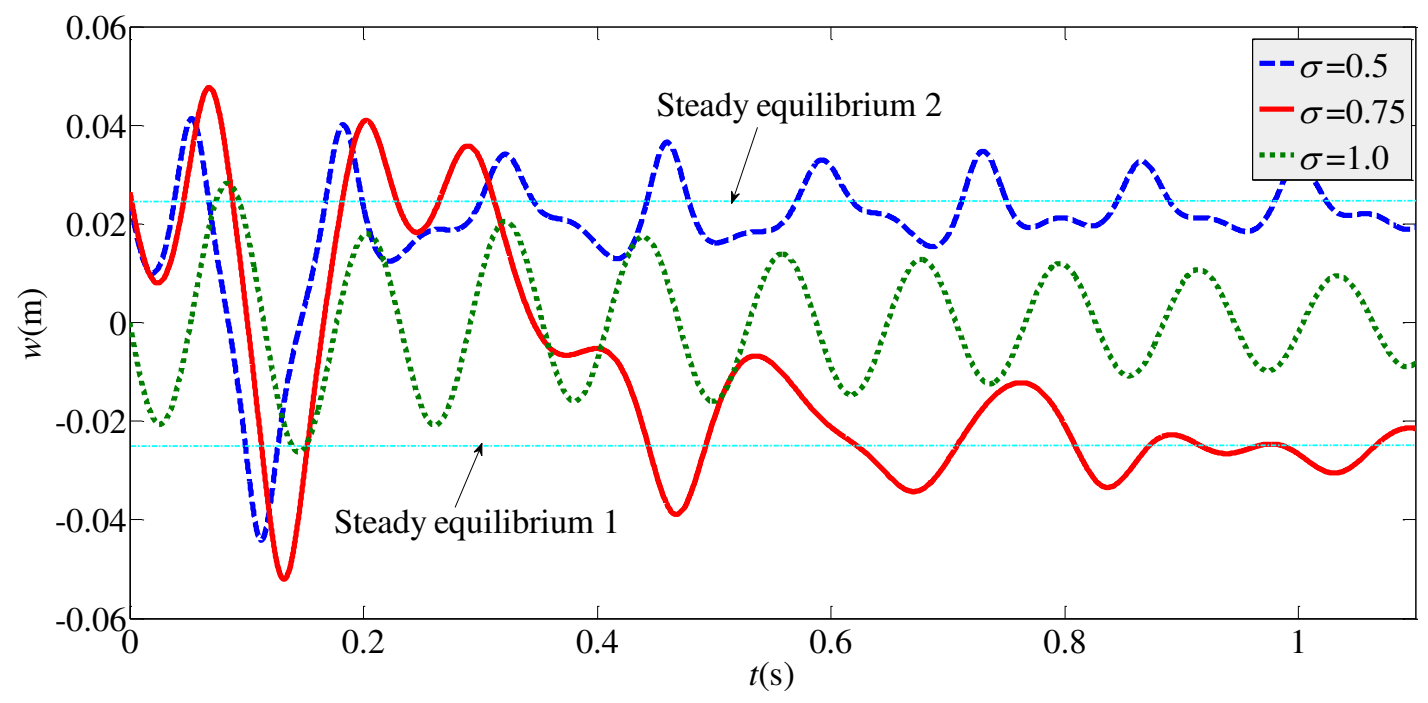

(b) The displacement time history of the NES system

Figure 9 The nonlinear motion behaviors of the primary system and NES system with a deterministic impulsive excitation $v_{0}=1.3232 \mathrm{~m} / \mathrm{s}$

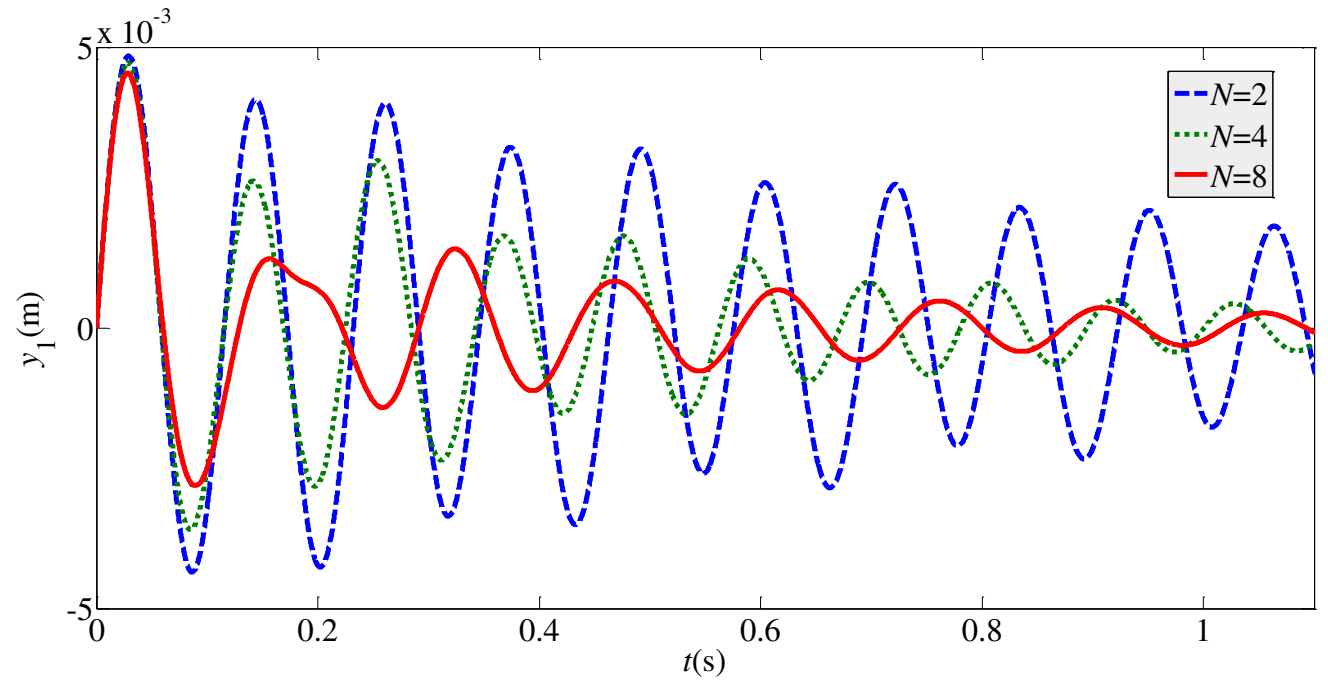

(a) The displacement time history of the primary system 


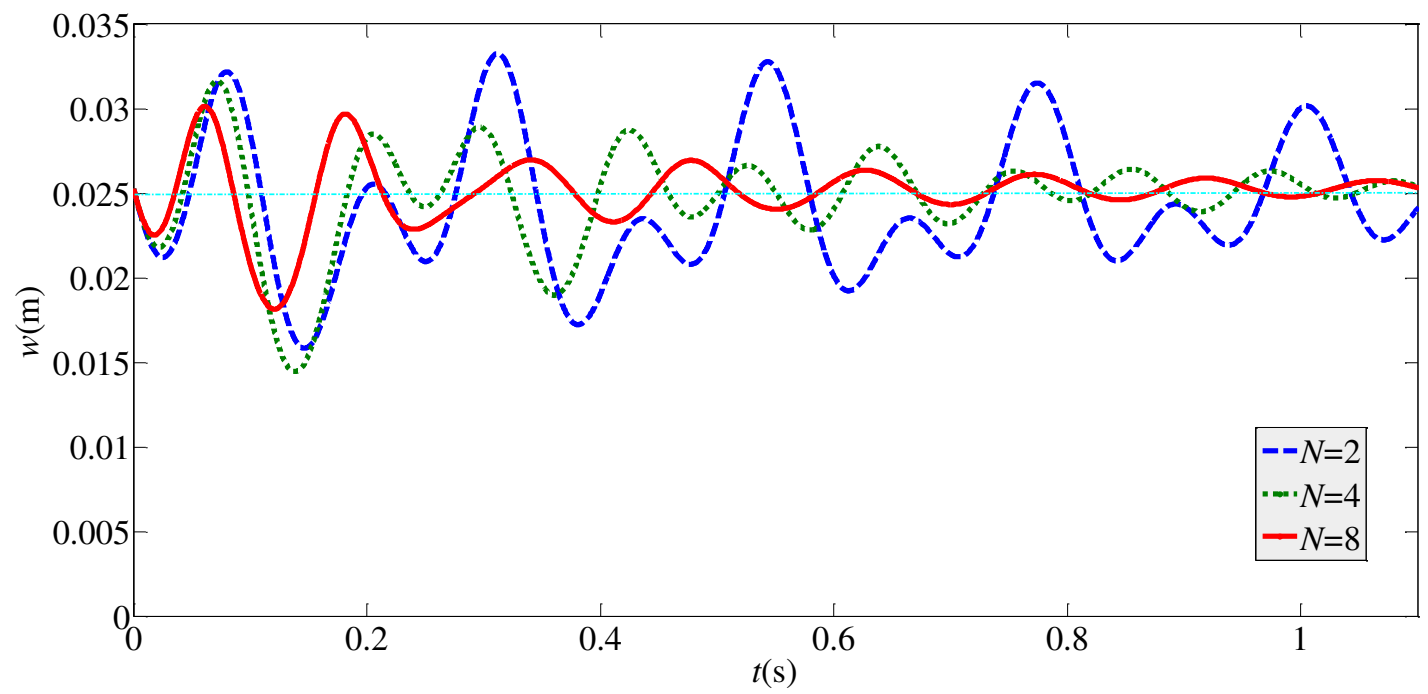

(b) The displacement time history of the NES system

Figure 10 The nonlinear motion behaviors of the primary system and NES system with different number of ECDVA cells $\left(v_{0}=0.2646 \mathrm{~m} / \mathrm{s}\right)$

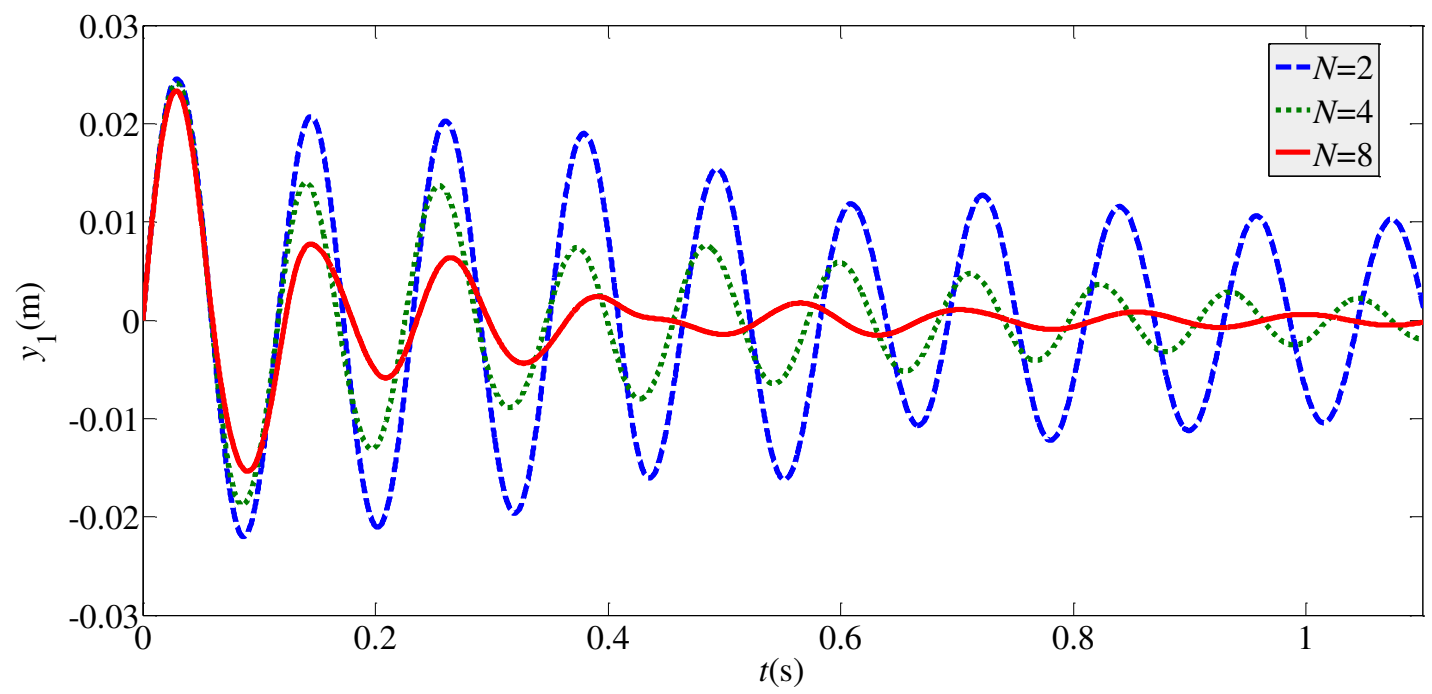

(a) The displacement time history of the primary system

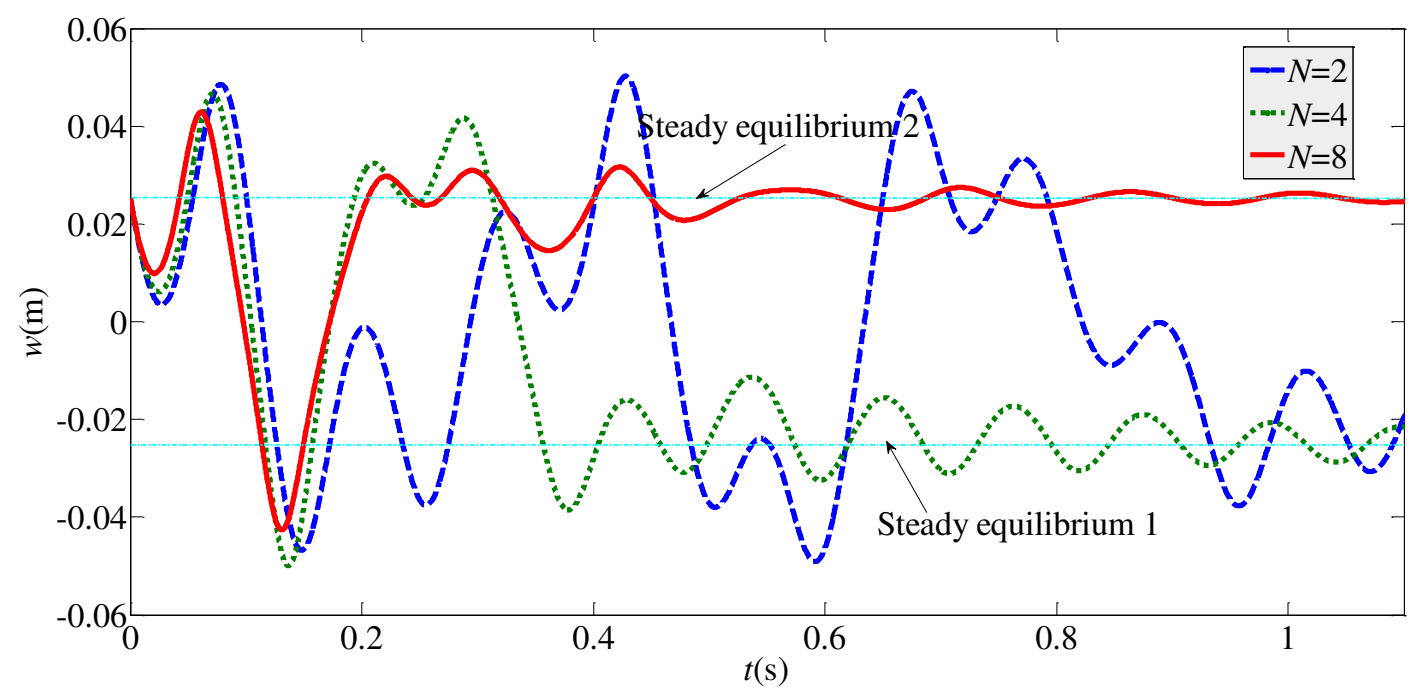

(b) The displacement time history of the NES system 
Figure 11 The nonlinear motion behaviors of the primary system and NES system with different number of EC-

$$
\text { DVA cells }\left(v_{0}=1.3232 \mathrm{~m} / \mathrm{s}\right)
$$

\subsection{The effects of EC-DVA number on the vibration suppression performance of clustered EC-DVAs}

An individual EC-DVA with constant mass may not possess satisfactory vibration absorption and energy conversion performance. However, assuming that the total attached mass is constant, the number of EC-DVAs can be modulated, and then an optimal number of EC-DVAs can be determined to obtain the optimal vibration absorption and energy conversion performance. In this section the effects of number of EC-DVAs on energy conversion efficiency and residual kinetic energy ratio are investigated with the application of a single impulse to the primary linear system. Hereby, the ratio of total clustered EC-DVAs mass and primary system mass is set to 0.2 . Figure 12 gives color plots of energy conversion efficiency $\eta_{\mathrm{h}}$ resulting from the application of a single impulse to the primary linear system, evaluated at $\tau=60$ as function of impact velocity $v_{0}$ and length ratio $\sigma$ with $\mu N=0.2$. From Figure 12 (a) it can be easily seen that chaotic motions including inter-well and in-well motions are dominant when the ambient input energy ranges from a small beginning into a mighty. Figure 12(b), 12(c) and 12(d) it can be found that stable inter-well motions are observed when the number of EC-DVAs increases, and with an appropriate range of length ratio $\sigma$ the energy conversion efficiency maintains an optimal level when $N$ is larger than 6 . From Figure 12 it can be also seen that the EC-DVA of small length ratio $\sigma$ performs not well in energy conversion even under large input energy conditions when the number of EC-DVAs increases. Moreover, it can be concluded in Figure 12 that when the length ratio $\sigma$ is less than 0.5 absolute in-well motions are easily seen in EC-DVA with low input energy, and chaotic motions are triggered with an appropriately large input energy. The phenomenon of the motion evolution is due to the matching of the minimum energy to overcome the potential barrier and the ambient input energy.

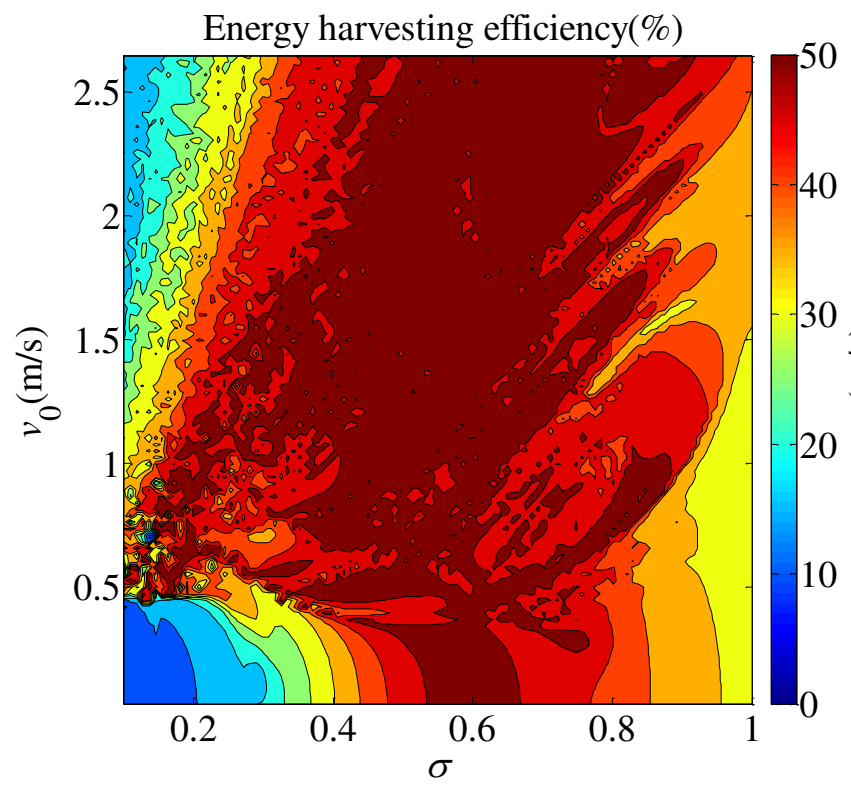

(a)

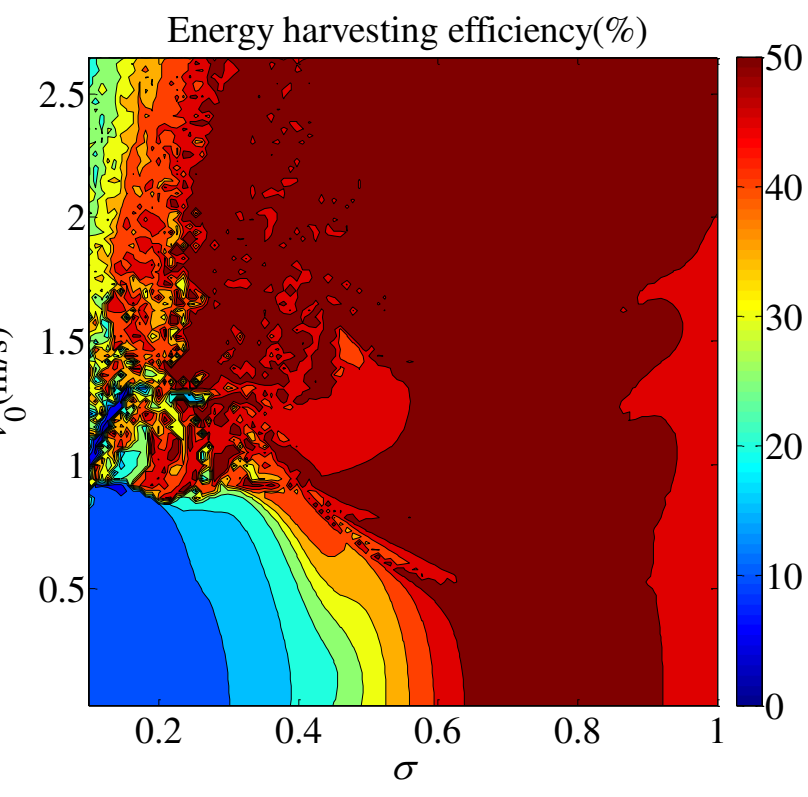

(b) 


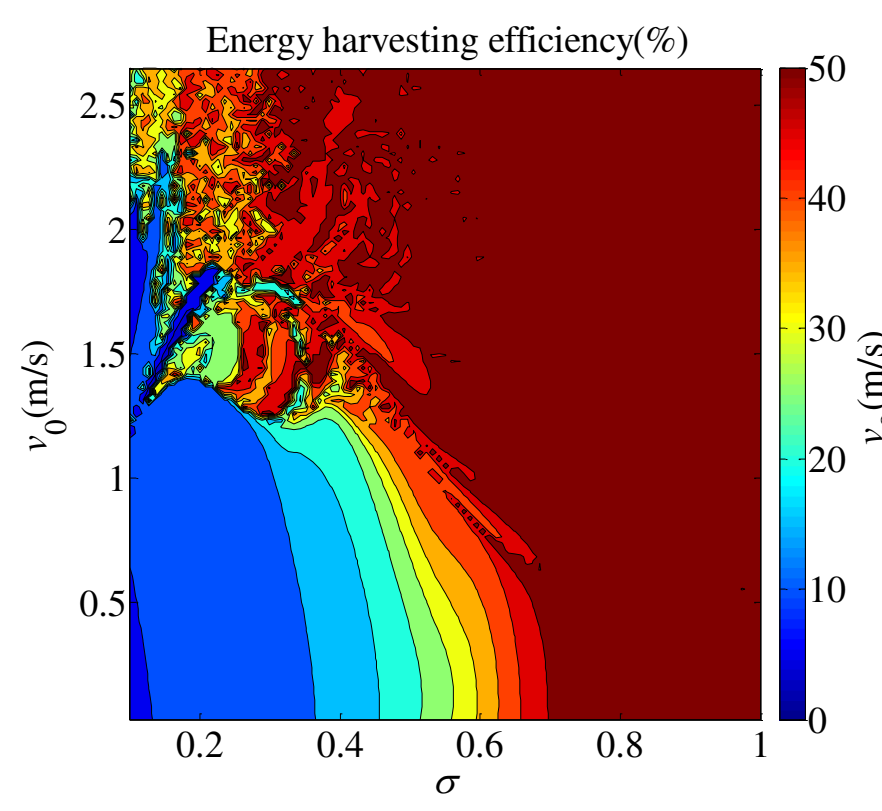

(c)

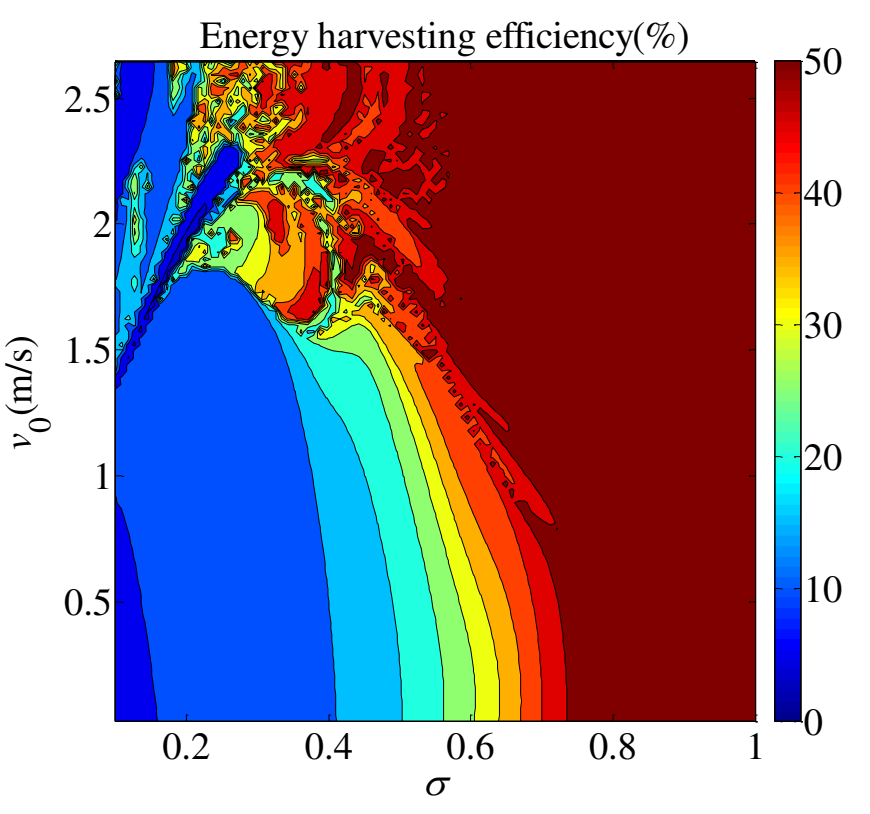

(d)

Figure 12 Color plots of energy conversion efficiency $\eta_{\mathrm{h}}$ resulting from the application of a single impulse to the primary linear system, evaluated at $\tau=60$ as function of impact velocity $v_{0}$ and length ratio $\sigma$ with $\mu N=0.2$. (a) $N=2$;

$$
\text { (b) } N=4 \text {; (c) } N=6 \text {; (d) } N=8
$$

Figure 13 presents color plots of residual kinetic energy ratio $\eta_{\mathrm{k}}$ resulting from the application of a single impulse to the primary linear system, evaluated at $\tau=60$ as function of impact velocity $v_{0}$ and length ratio $\sigma$ with $\mu N=0.2$. From Figure 13(a), 13(b), 13(c) and 13(d) it can be obviously found that the residual kinetic energy of the primary system excited by large impulsive excitations decreases with the increase in number of EC-DVAs, and the vibration absorption performance of the system with large length ratio $\sigma(\sigma>0.6)$ is excellent with the increase in number of EC-DVAs. However, the vibration absorption performance of the system with small length ratio $\sigma$ is more and more unsatisfactory when number of EC-DVAs increases. Thereby, a compromise in number of EC-DVAs should be made to obtain the optimal vibration absorption performance, consequently the solution for determining the optimal number of EC-DVAs is meaningful for vibration suppression.

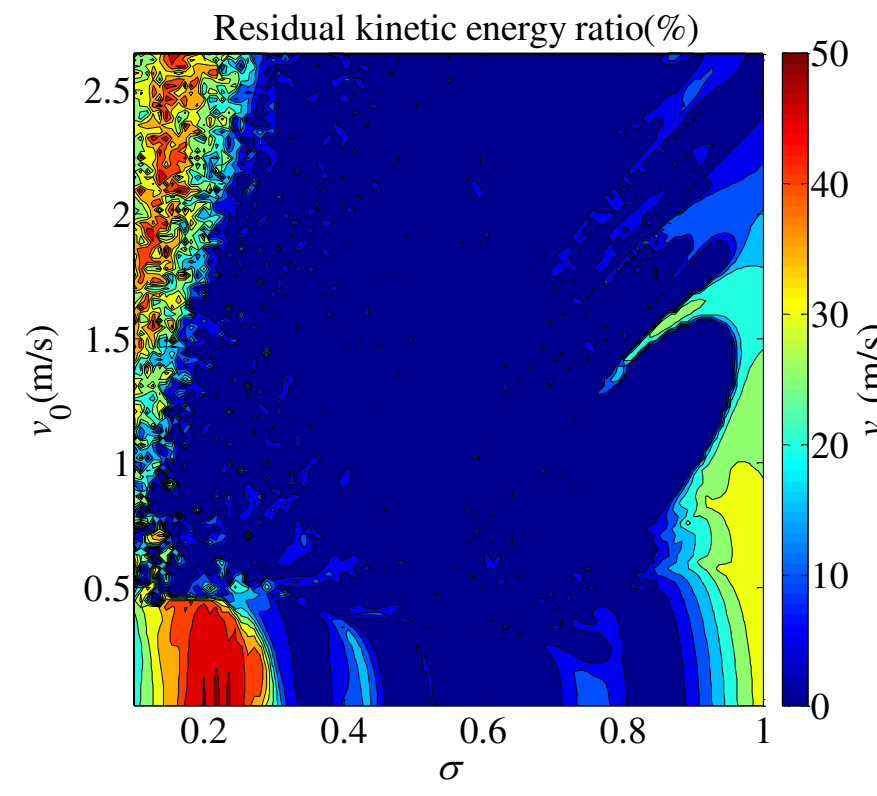

(a)

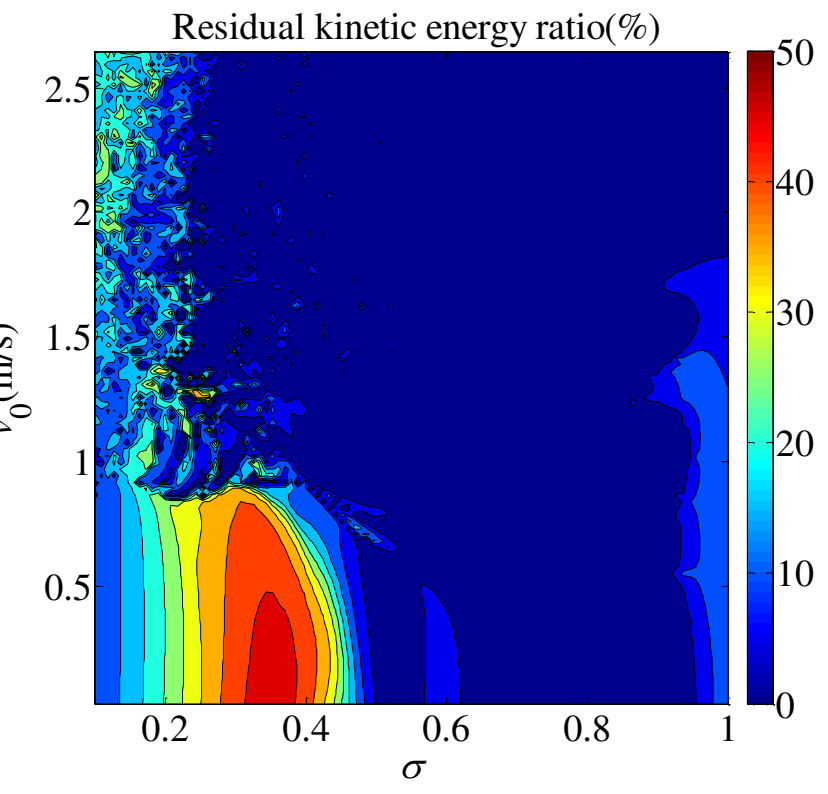

(b) 


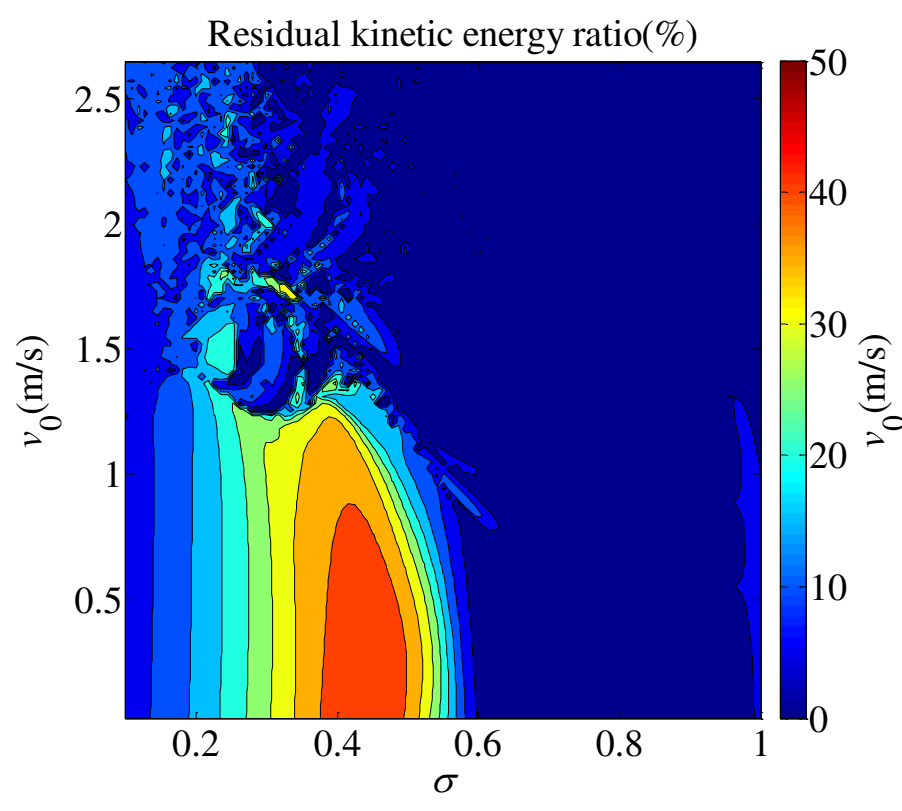

(c)

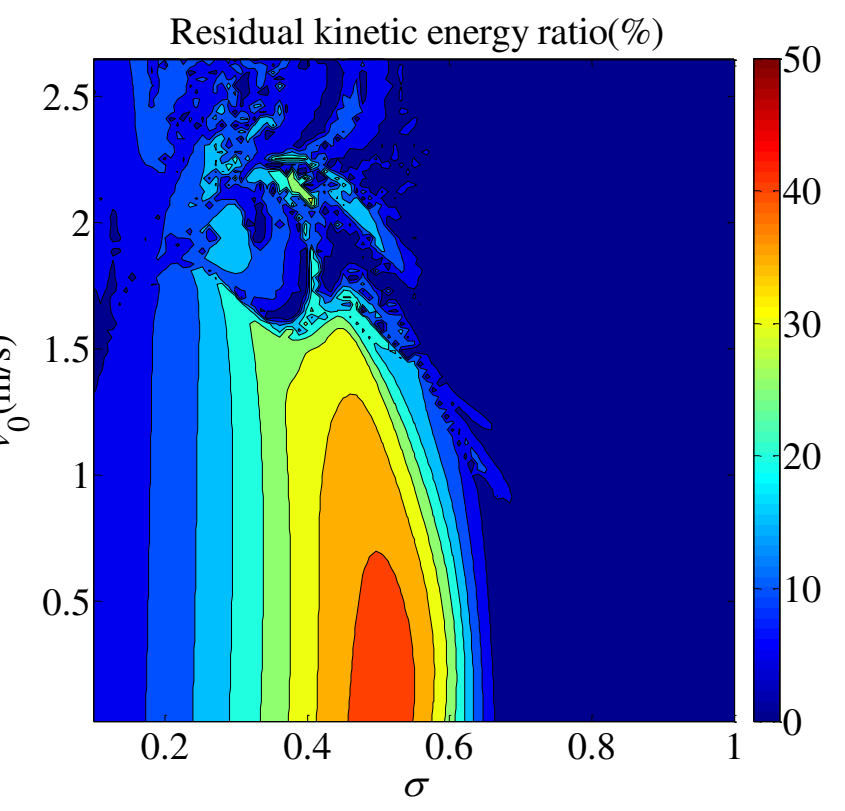

(d)

Figure 13 Color plots of residual kinetic energy ratio $\eta_{\mathrm{k}}$ resulting from the application of a single impulse to the primary linear system, evaluated at $\tau=60$ as function of impact velocity $v_{0}$ and length ratio $\sigma$ with $\mu N=0.2$. (a) $N=2$; (b) $N=4$; (c) $N=6$; (d) $N=8$

In order to understand the impulse response of the primary system attached clustered EC-DVAs of different number, wavelet transform and phase portrait are employed to observe vibration absorption performance of the system and the snap-through motions. In this section the calculation parameters are set as $\mu N=0.2, \sigma=0.75$, $v_{0}=2.65 \mathrm{~m} / \mathrm{s}$. Figure 14 shows the displacement wavelet spectra of primary system with different number of EC-DVAs. From Figure 14 it can be seen that approximate $10 \mathrm{~Hz}$ frequency response component of the primary system is gradually decreased by increasing the number of EC-DVAs, and the vibration suppression performance is the most remarkable when the number of EC-DVAs is set to 6. From Figure 14(d) it can be found that vibration response of the primary system is increasingly enhanced when the number of EC-DVAs is set to 8 . Therefore, determining an optimal number of EC-DVAs is helpful for improving the vibration suppression performance.

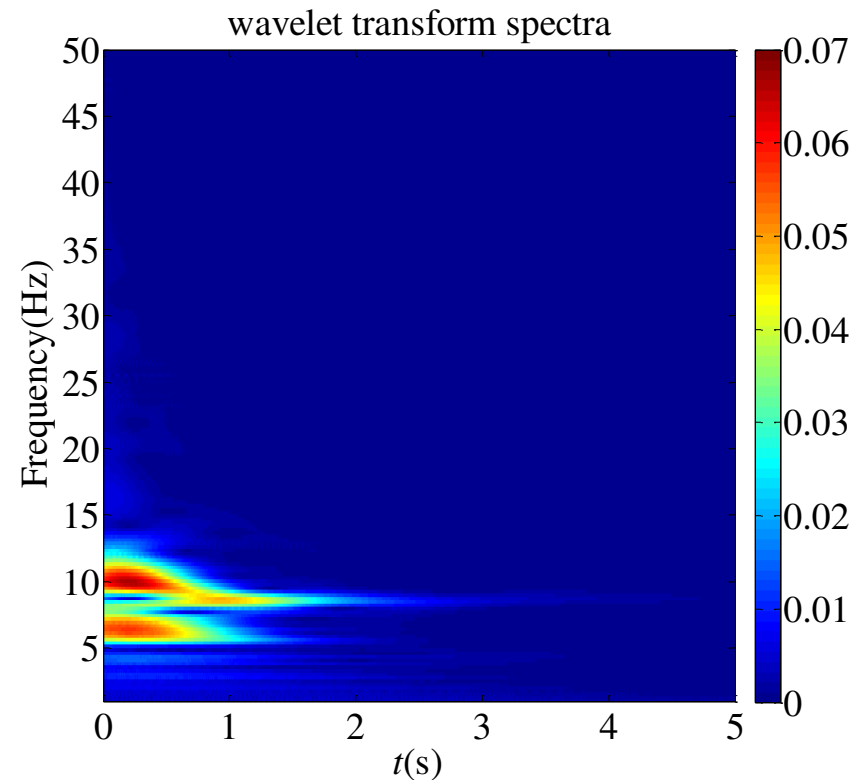

(a)

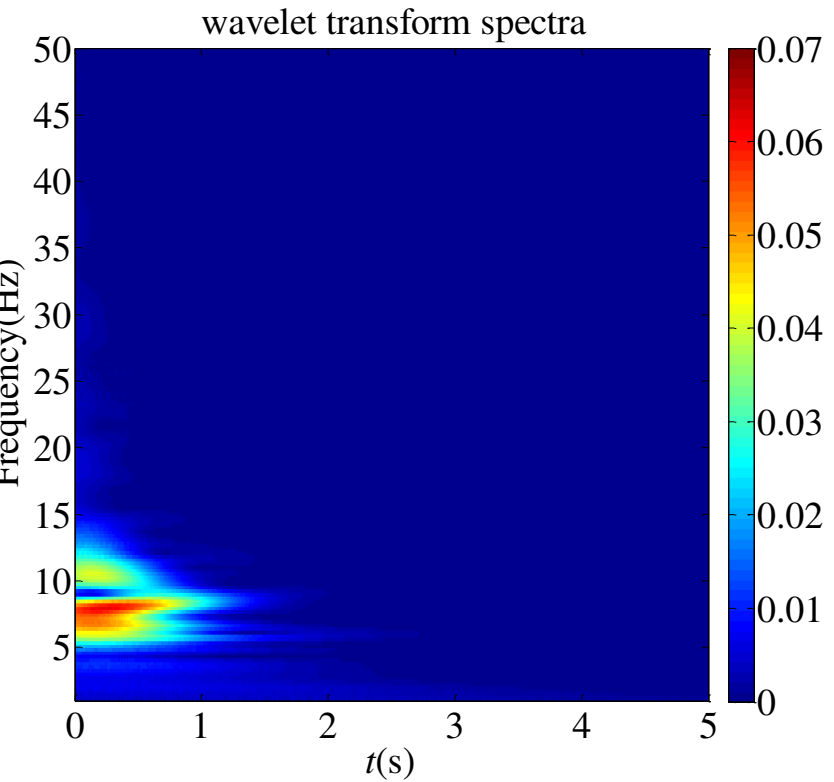

(b) 


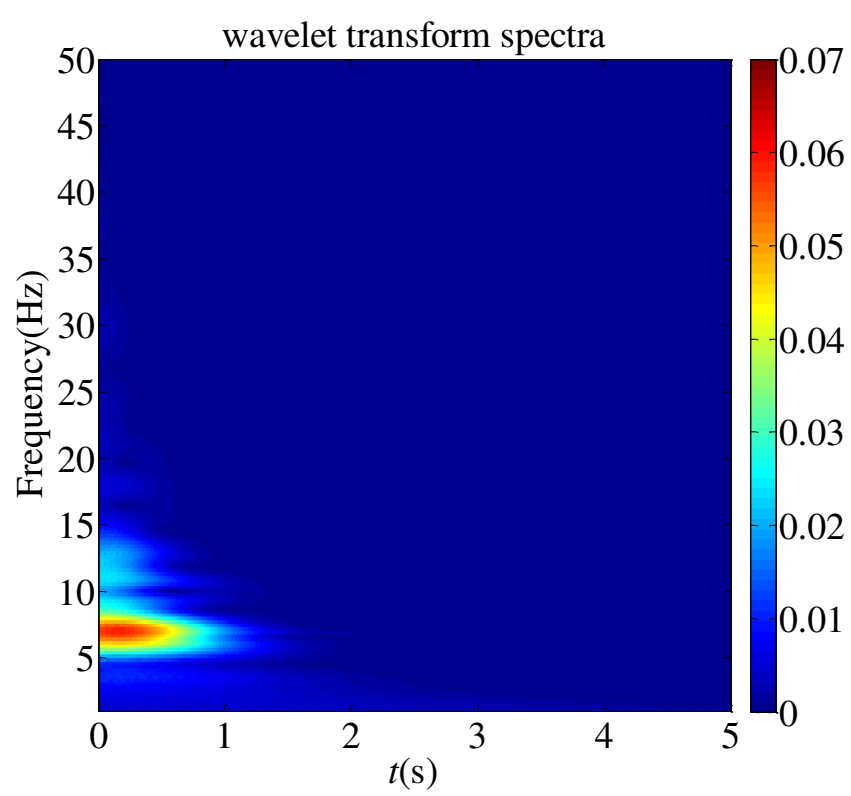

(c)

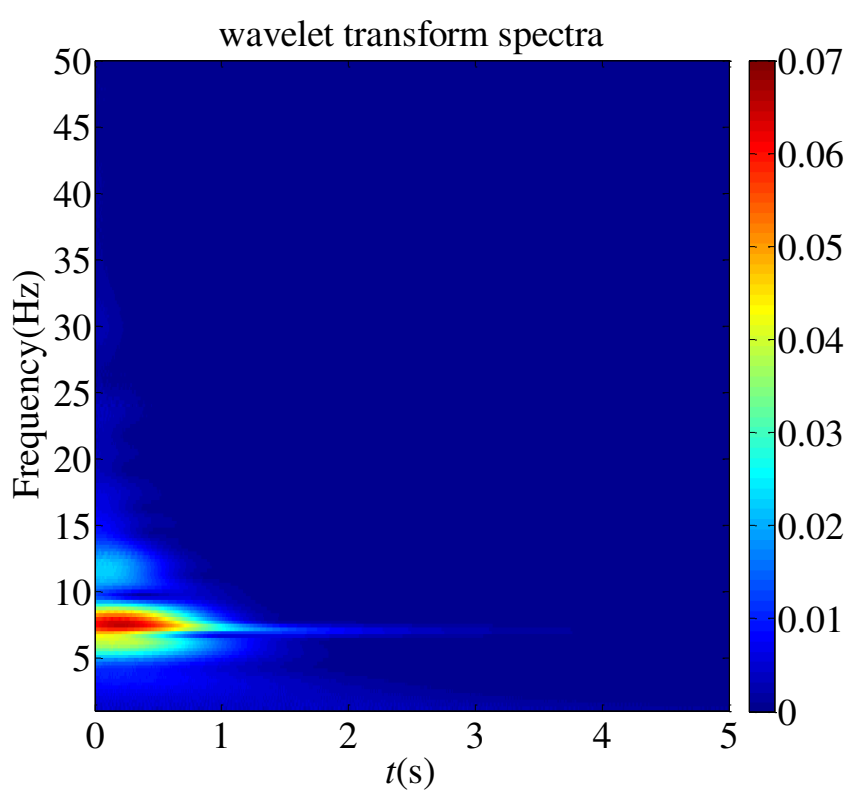

(d)

Figure 14 The displacement wavelet spectra of primary system with different number of EC-DVAs $(\mu N=0.2$,

$$
\sigma=0.75, v_{0}=2.65 \mathrm{~m} / \mathrm{s} \text { ). (a) } N=2 \text {; (b) } N=4 \text {; (c) } N=6 \text {; (d) } N=8
$$

The motion of clustered EC-DVAs can be used to account for the impulse response of the primary system because the total energy is constant. Figure 15 presents the phase portrait of the uniform EC-DVA with different number of EC-DVAs, from which it can be easily found that in-well motions develop into the mixed chaos composed of in-well and cross-well motions. When the number of EC-DVAs increases to 6, the absolute cross-well motions form and bi-stable energy conversion mechanism is activated. Thereby, stable bi-stable snap-through motions can maximize the vibration suppression performance.

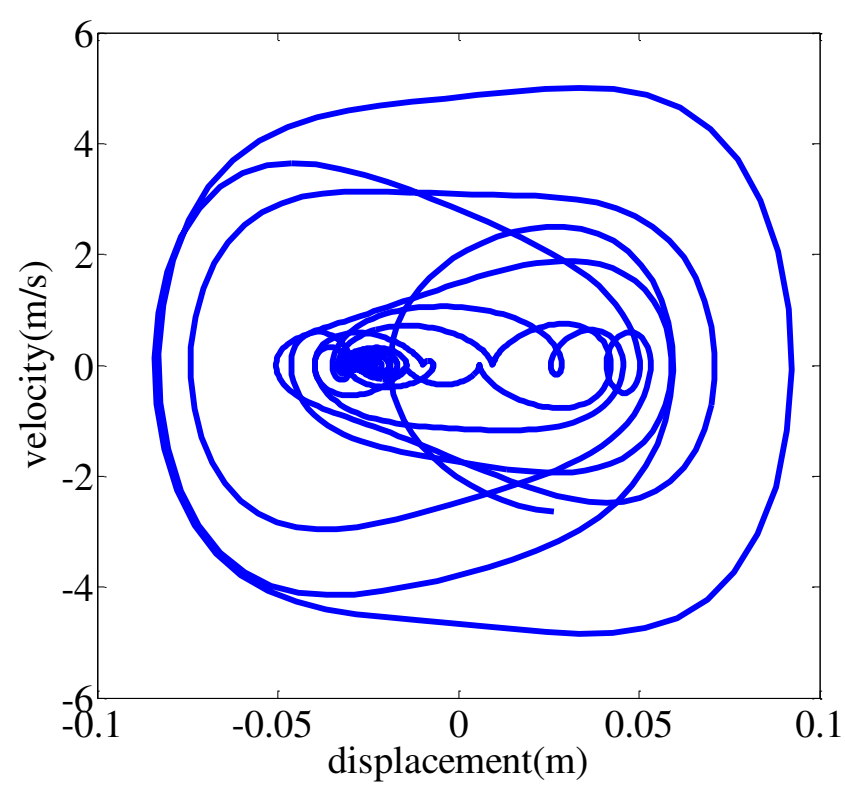

(a)

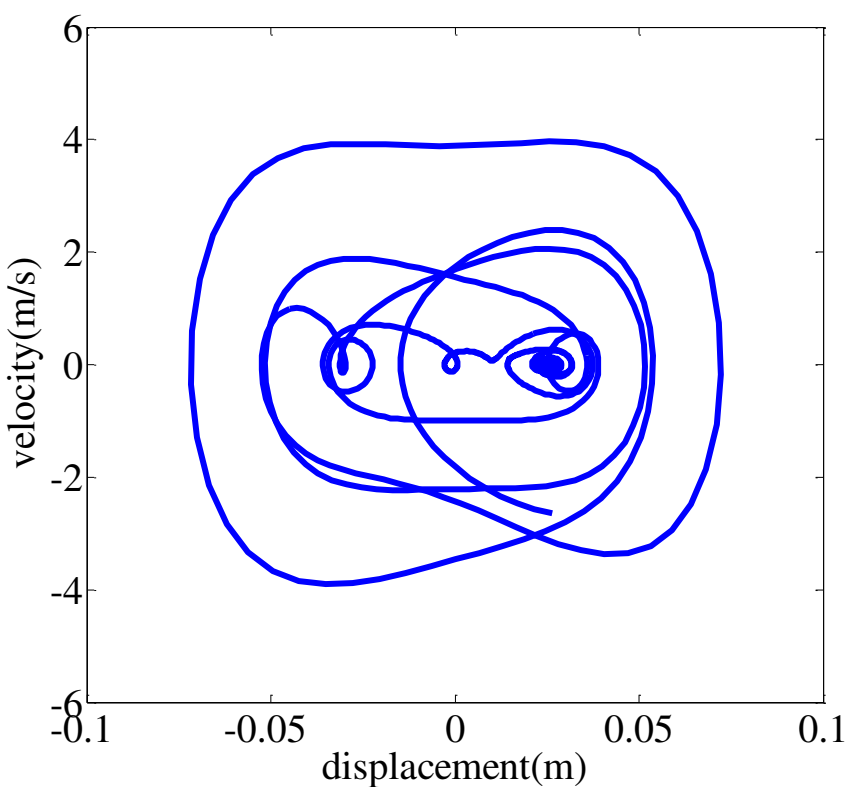

(b) 


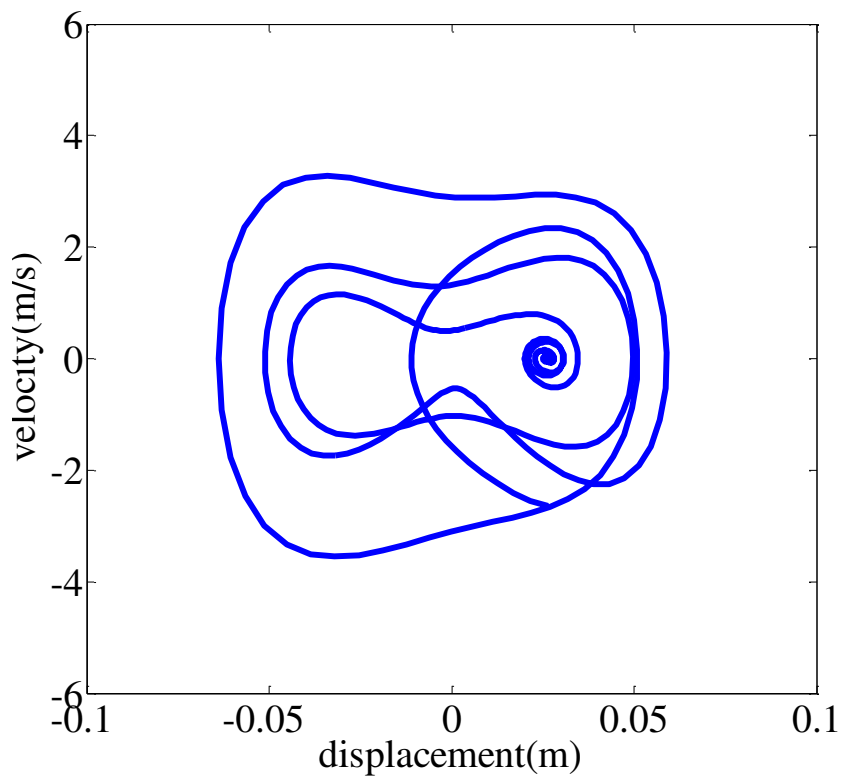

(c)

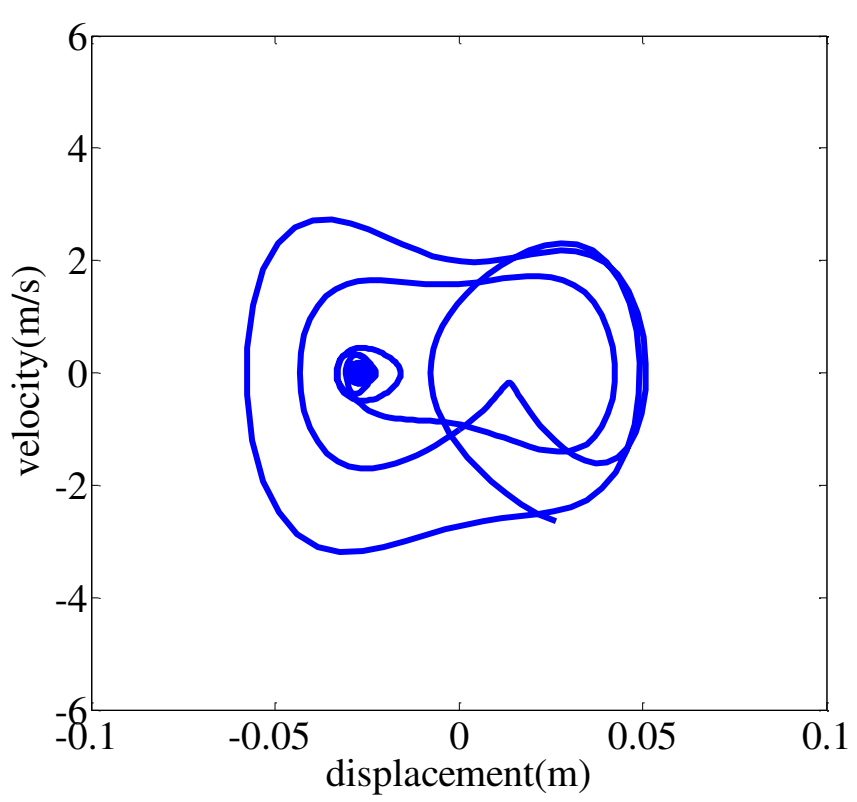

(d)

Figure 15 The phase portrait of the uniform EC-DVA with different number of EC-DVAs $(\mu N=0.2, \sigma=0.75$,

$$
v_{0}=2.65 \mathrm{~m} / \mathrm{s} \text { ). (a) } N=2 \text {; (b) } N=4 \text {; (c) } N=6 \text {; (d) } N=8
$$

For the sake of well understanding the evolution from monostable to bi-stable when the system is applied to low input energy, different number of EC-DVAs are considered to investigate the influence of number of EC-DVAs on the implementation of bi-stable system. Herein, the calculation parameters are set to $\mu N=0.2, \sigma=0.75, v_{0}=0.53 \mathrm{~m} / \mathrm{s}$. Figure 16 gives the displacement wavelet spectra of the primary system with different number of EC-DVAs, from which it can be evidently seen that vibration absorption of the primary system $(N=4)$ is much more significant than its counterpart $(N=8)$. Figure 17 shows the phase portrait of the uniform EC-DVA with different number of EC-DVAs. From Figure 17 it can be found that the motions of EC-DVA cell $(N=8)$ are almost in-well motion, and the motions of EC-DVA cell $(N=4)$ are the chaotic motions including in-well and cross-well motions that indicate the potential barrier is overcome to realize bi-stability.

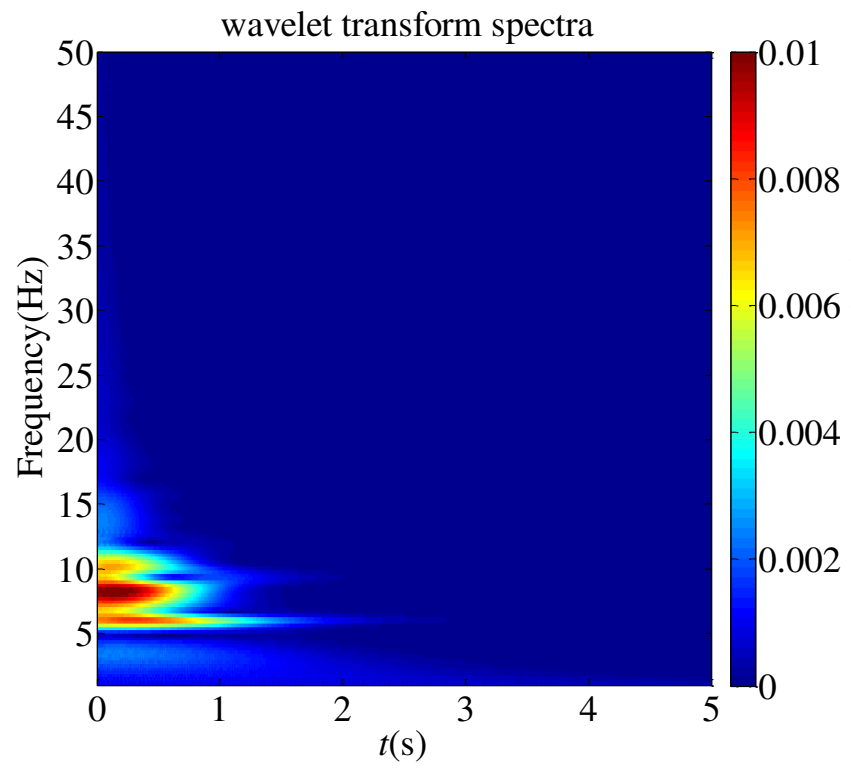

(a)

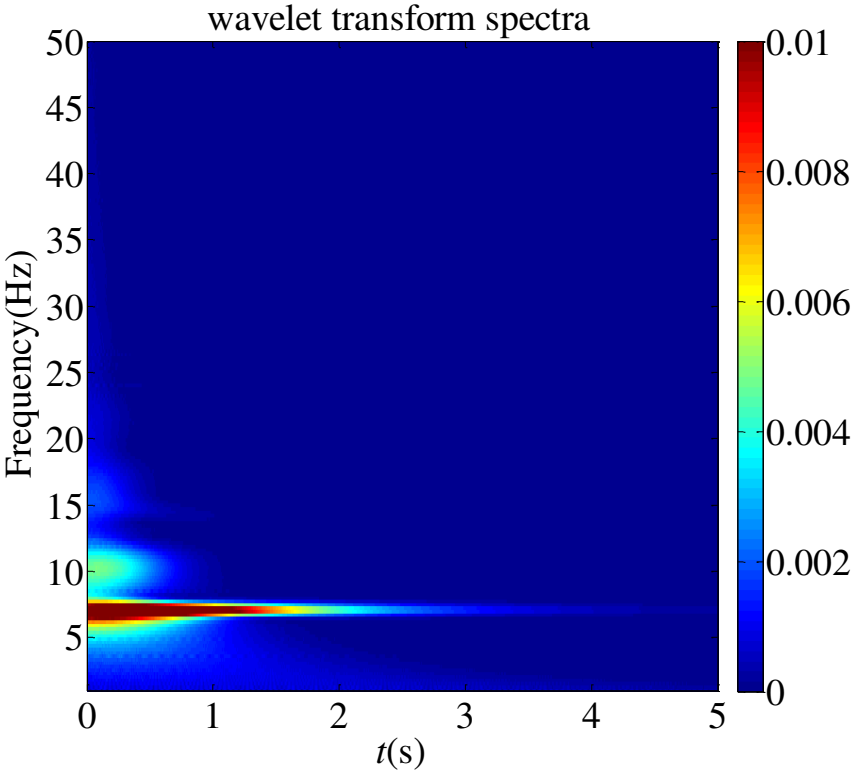

(b)

Figure 16 The displacement wavelet spectra of primary system with different number of EC-DVAs $(\mu N=0.2$, 


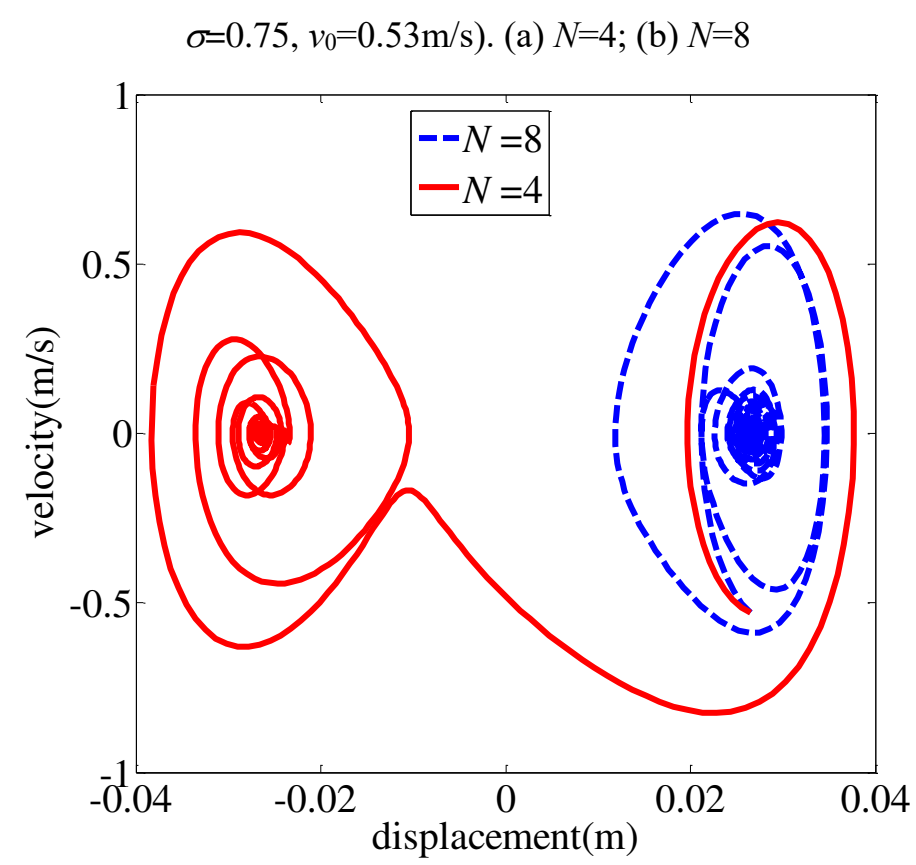

Figure 17 The phase portrait of the uniform EC-DVA with different number of EC-DVAs ( $\mu N=0.2, \sigma=0.75$,

$$
v_{0}=0.53 \mathrm{~m} / \mathrm{s} \text { ). (a) } N=4 \text {; (b) } N=8
$$

5.3 Optimization strategies of vibration absorption and energy conversion performance of clustered EC-DVAs

5.3.1 The optimal vibration absorption and energy conversion performance based on length ratio optimization

In this section the objective function is defined as the maximum energy conversion efficiency or the minimum residual kinetic energy ratio. The optimization strategy (described in Figure 4 and Figure 5) is to modulate the length ratio $\sigma$ according to the change of ambient input energy to obtain the desired objective function. The control group is composed of an inflexible bi-stable system $(\sigma=0.75)$ and a pure nonlinear energy sink (NES). Hereby, the ratio of total clustered EC-DVAs mass and primary system mass is set to 0.2 . Figure 18 presents the calculated energy conversion efficiency $\eta_{\mathrm{h}}$ resulting from the application of a single impulse to the primary linear system, evaluated at $\tau=60$ as function of impact velocity $v_{0}$ with $\mu N=0.2$. From Figure 18 it can be easily found that the energy conversion efficiency after optimization is remarkably larger than its counterpart of the NES, additionally, it can be surprisingly found that an inflexible bi-stable system $(\sigma=0.75)$ can induce almost identical energy conversion efficiency compared with the optimal case when the number of EC-DVAs is appropriate. From Figure 18(d) it can be easily found that the defects of an inflexible bi-stable system are exposed, remarkable fluctuations and reduction of energy conversion efficiency are seen in an inflexible bi-stable system when the number of EC-DVAs is 8 . The energy conversion efficiency caused by a pure NES increases with the increase in the number of EC-DVAs, however, the energy conversion efficiency after dynamic optimization is independent on the number of EC-DVAs and approximately wanders around $54 \%$. 


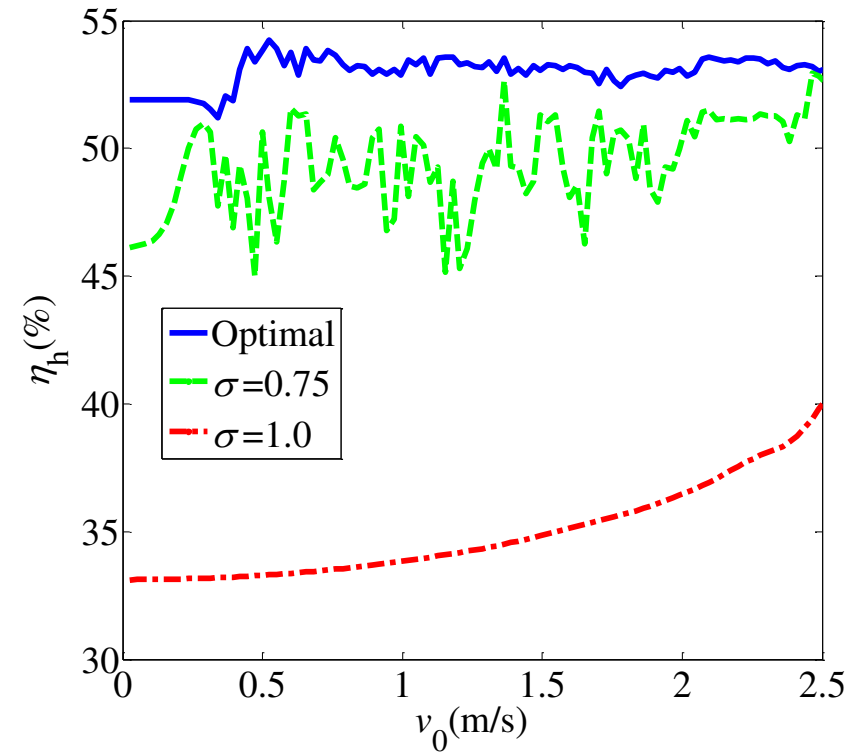

(a)

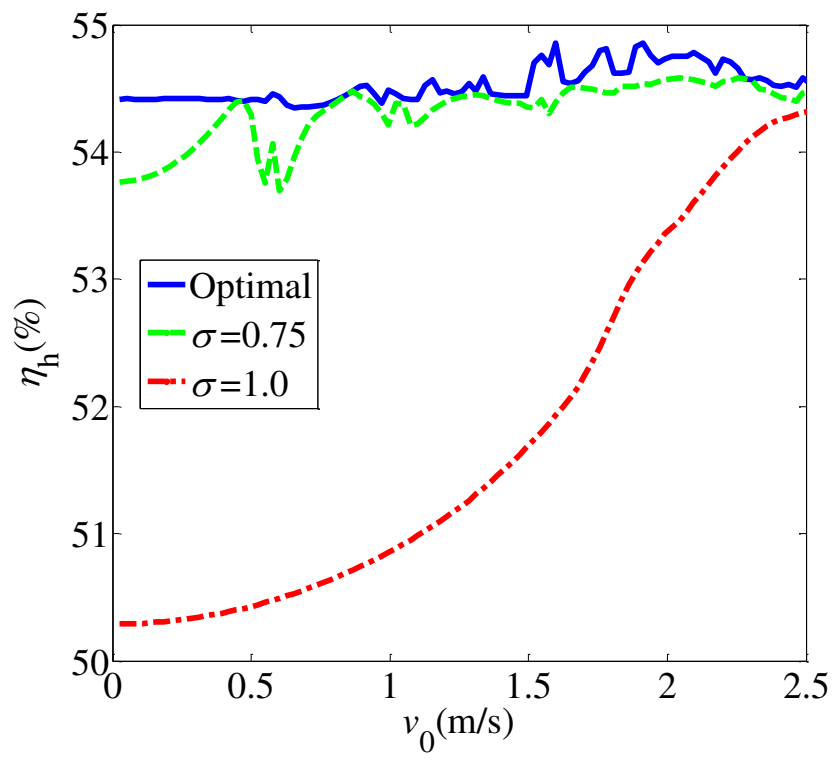

(c)

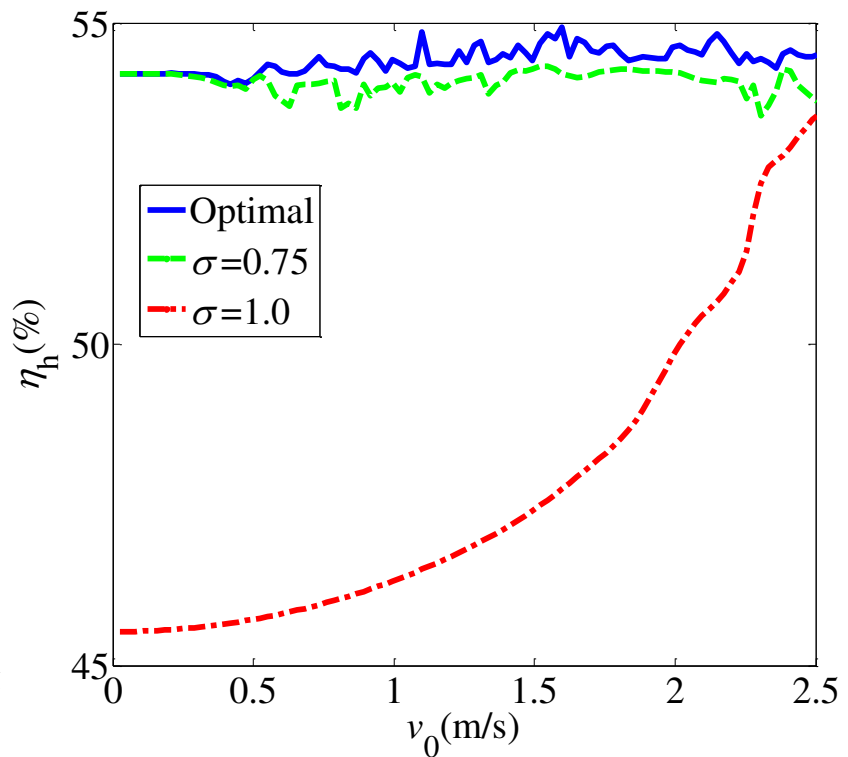

(b)

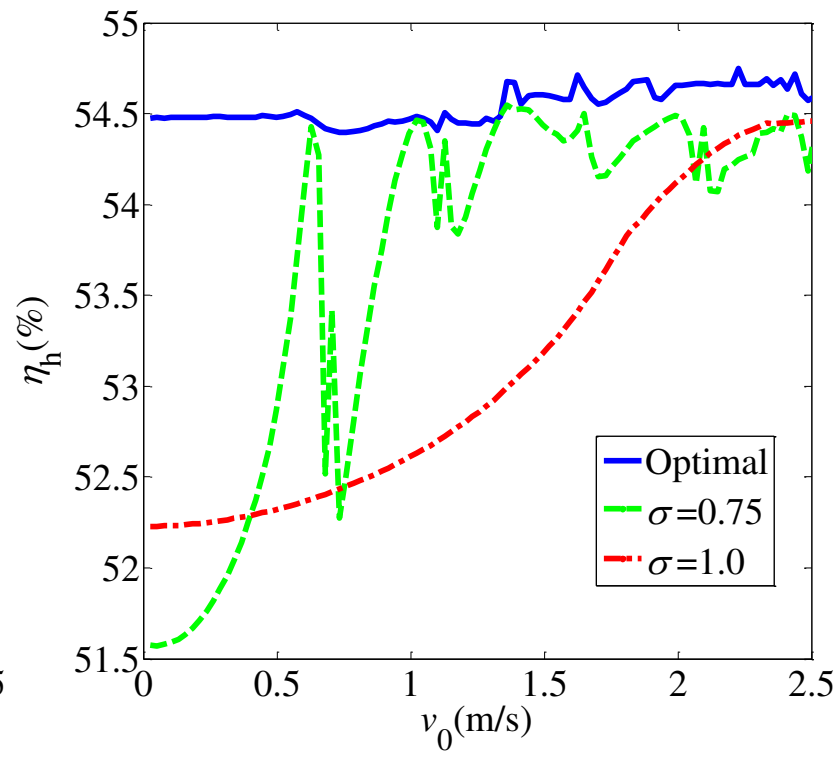

(d)

Figure 18 The calculated energy conversion efficiency $\eta_{\mathrm{h}}$ resulting from the application of a single impulse to the primary linear system, evaluated at $\tau=60$ as function of impact velocity $v_{0}$ with $\mu N=0.2$. (a) $N=2$; (b) $N=4$; (c) $N=6$; (d) $N=8$

The optimal length ratio for the maximum energy conversion efficiency $\eta_{\mathrm{h}}$ with $\mu N=0.2$ is presented in Figure 19. From Figure 19(a) it can be found that in order to achieve the maximum energy conversion efficiency the adjustment amplitude of length ratio is large (nearly from 0.1 to 0.8 ). With the increase in the number of EC-DVAs the tuned range of length ratio is decreased down, as shown in Figure 19(b), 19(c) and 19(d). Therefore, an appropriate number of EC-DVAs should be determined to facilitate the real-time modulation of length ratio. 


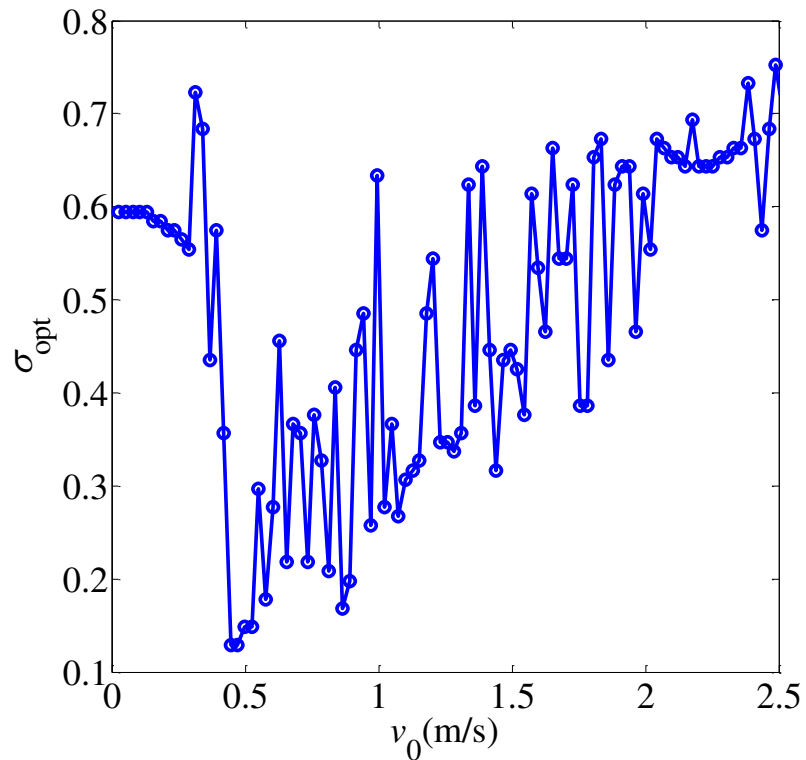

(a)

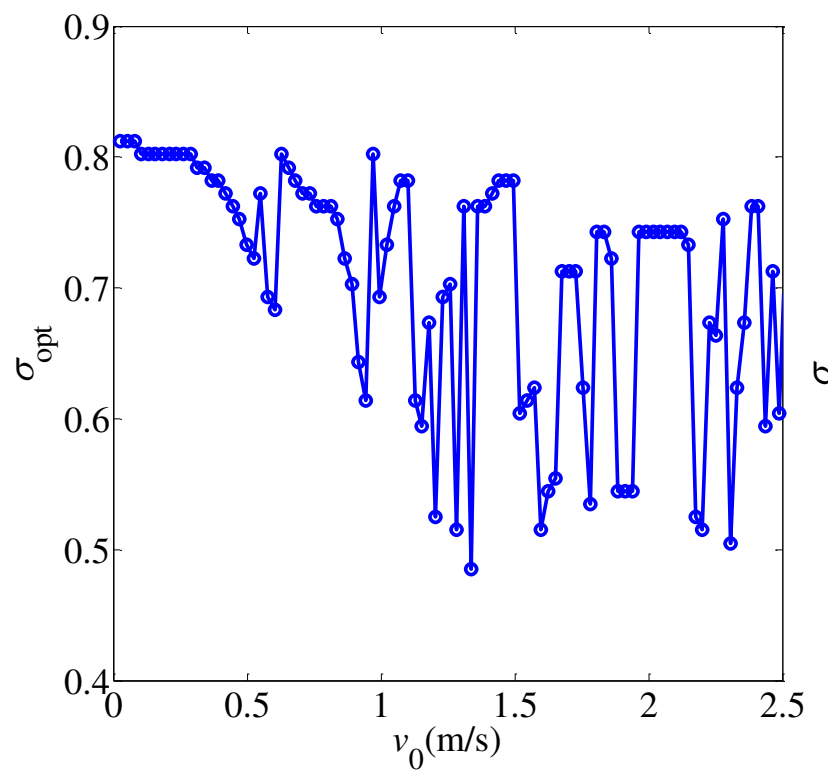

(c)

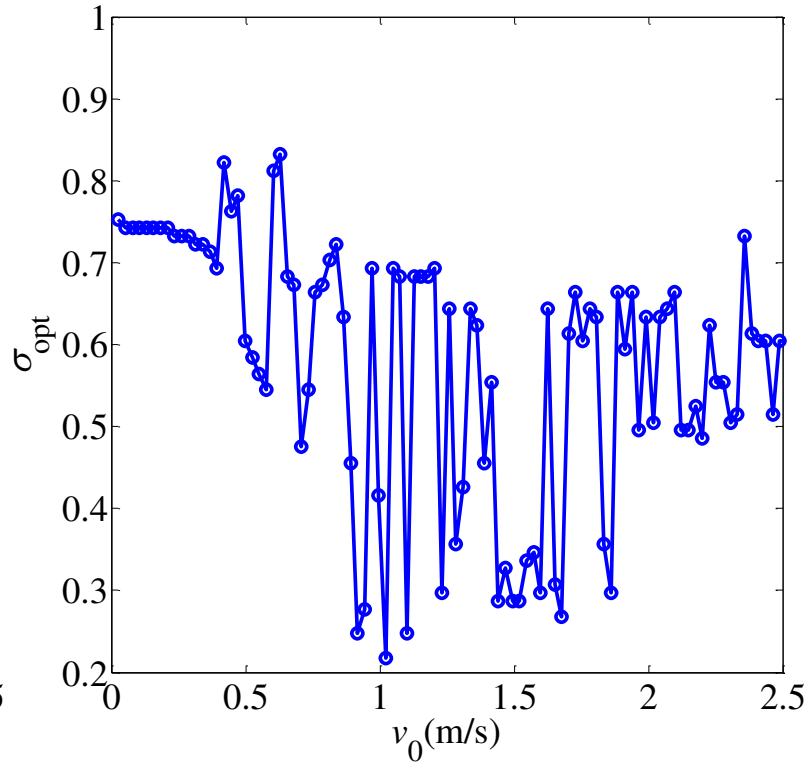

(b)

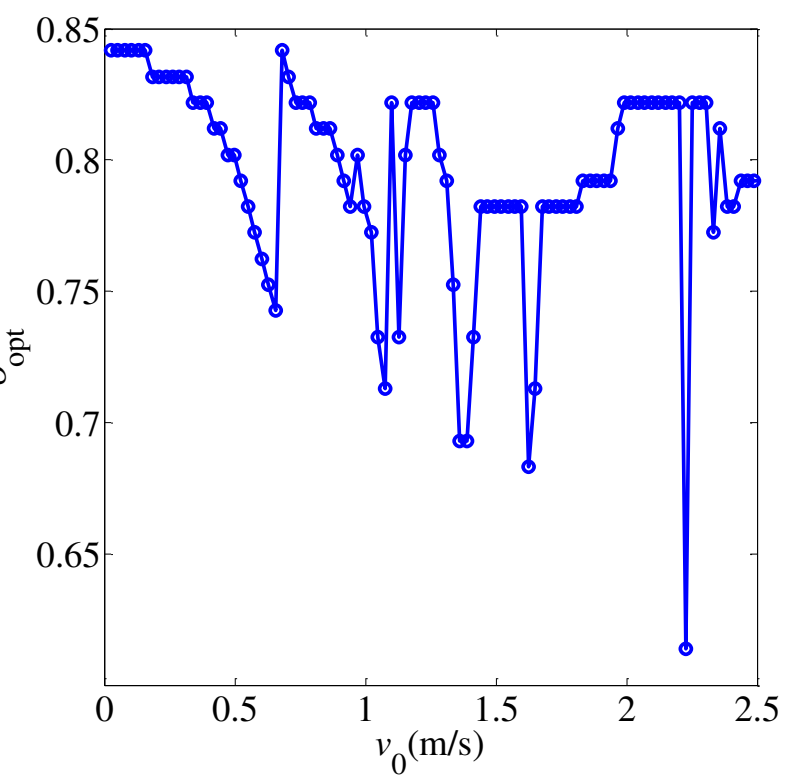

(d)

Figure 19 The optimal length ratio for the maximum energy conversion efficiency $\eta_{\mathrm{h}}$ with $\mu N=0.2$. (a) $N=2$; (b)

$$
N=4 \text {; (c) } N=6 \text {; (d) } N=8
$$

The alternative method to evaluate the vibration absorption performance is calculating the residual kinetic energy ratio of the primary system after a period of time. Figure 20 shows the calculated residual kinetic energy ratio $\eta_{\mathrm{k}}$ resulting from the application of a single impulse to the primary linear system, evaluated at $\tau=60$ as function of impact velocity $v_{0}$ with $\mu N=0.2$. From Figure 20 it can be seen that the calculated results are in good agreement with Figure 18. When the number of EC-DVAs is too small or too large, the residual kinetic energy ratio induced by an inflexible bi-stable system $(\sigma=0.75)$ is unsatisfactory, meanwhile, the residual kinetic energy ratio caused by a pure NES is reduced greatly with the increase in number of EC-DVAs. From Figure 20 it can be easily seen that the residual kinetic energy ratio after optimizing length ratio is approximately zero and independent on the number of EC-DVAs. 


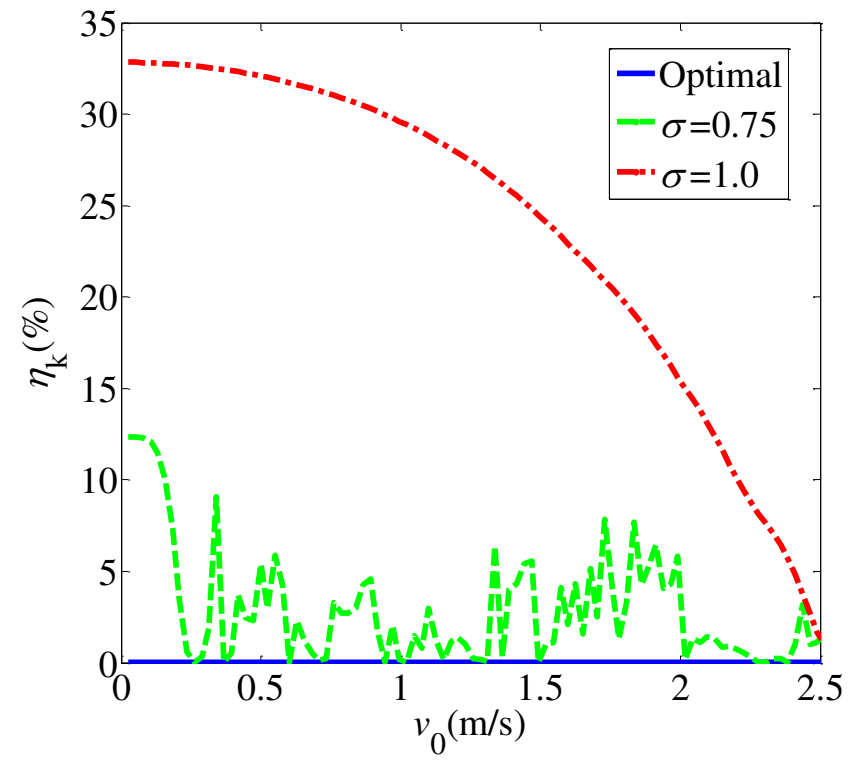

(a)

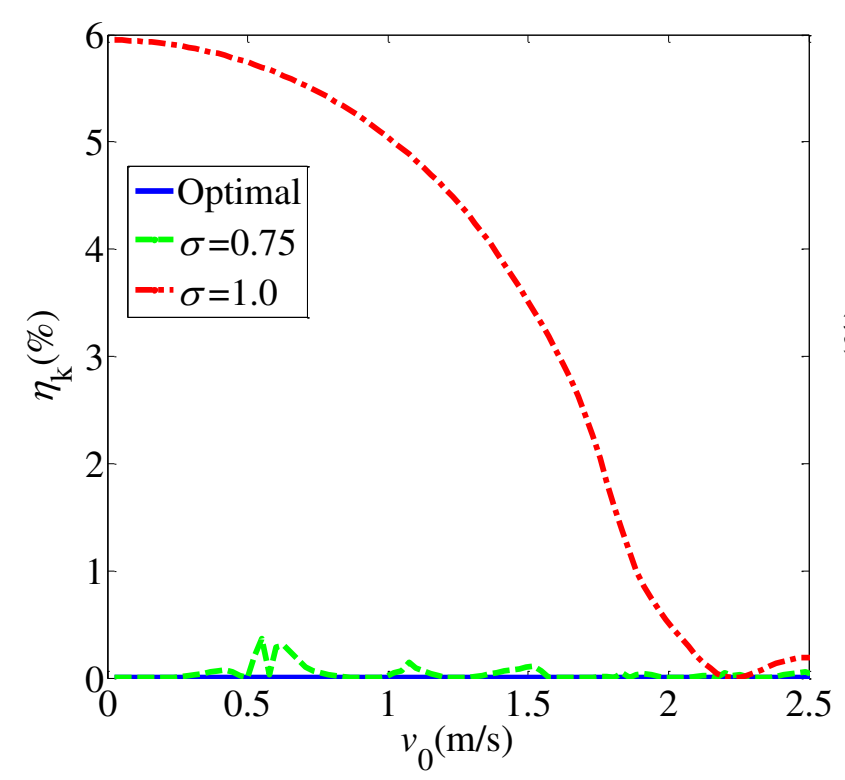

(c)

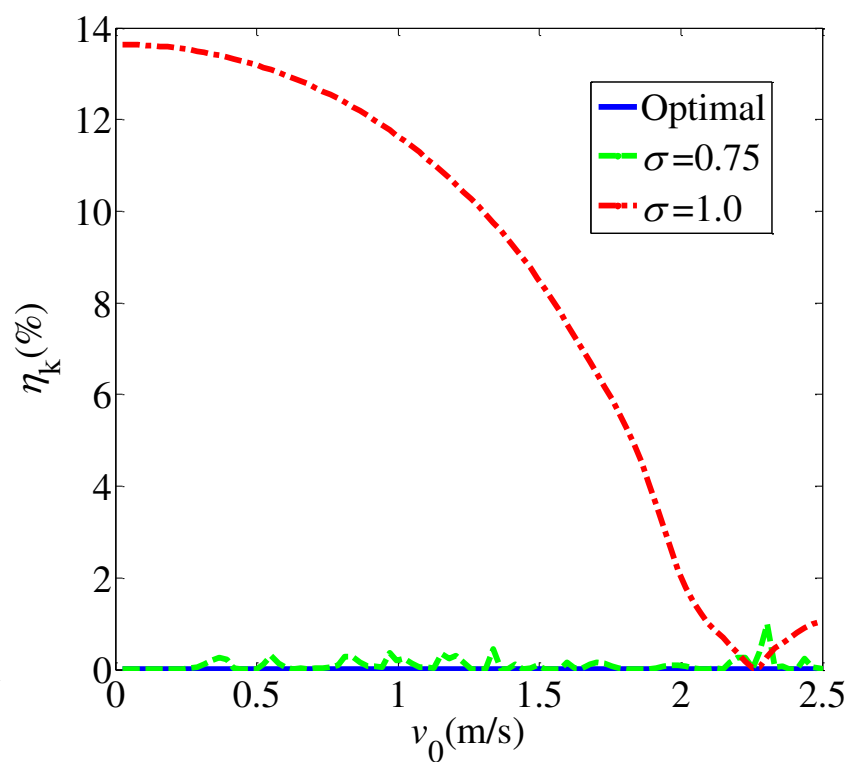

(b)

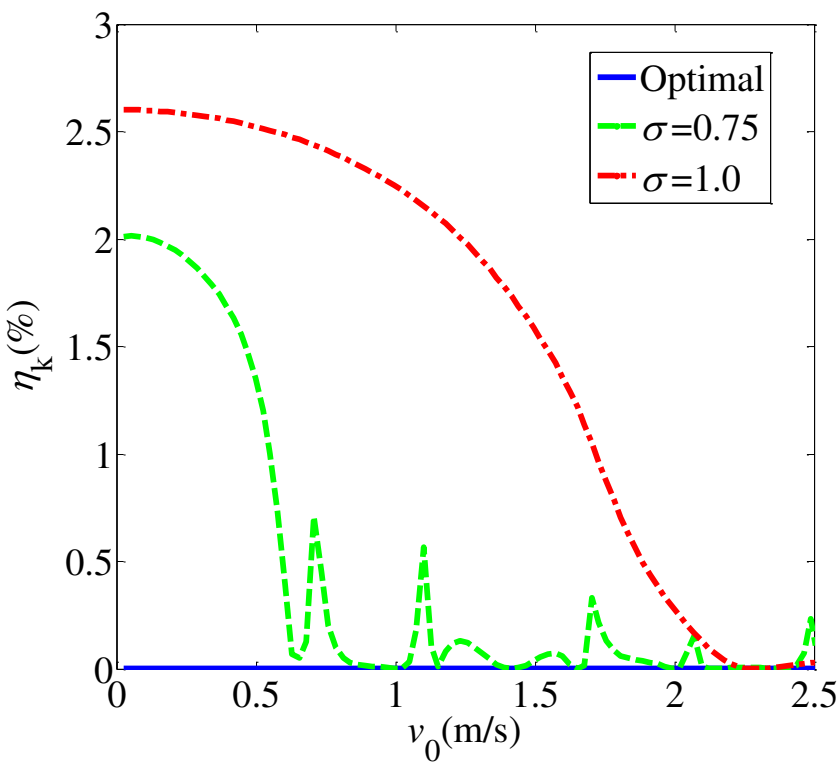

(d)

Figure 20 The calculated residual kinetic energy ratio $\eta_{\mathrm{k}}$ resulting from the application of a single impulse to the primary linear system, evaluated at $\tau=60$ as function of impact velocity $v_{0}$ with $\mu N=0.2$. (a) $N=2$; (b) $N=4$; (c) $N=6$; (d) $N=8$

Figure 21 describes the optimal length ratio for the minimum residual kinetic energy ratio $\eta_{\mathrm{k}}$ with $\mu N=0.2$. From Figure 21 it can be also seen that the fluctuation of optimal length ratio is remarkable when the number of EC-DVAs is small, inversely, the fluctuation of optimal length ratio is narrow when the number of EC-DVAs is large. Therefore, an appropriate number of EC-DVAs is indispensable for efficiently tuning the optimal length ratio to obtain the minimum residual kinetic energy ratio. 


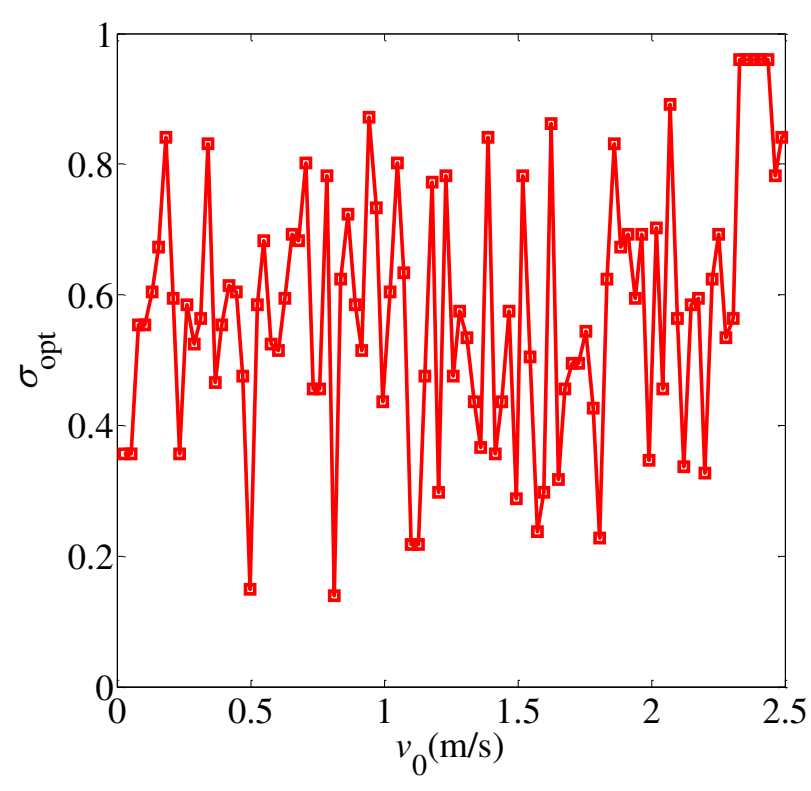

(a)

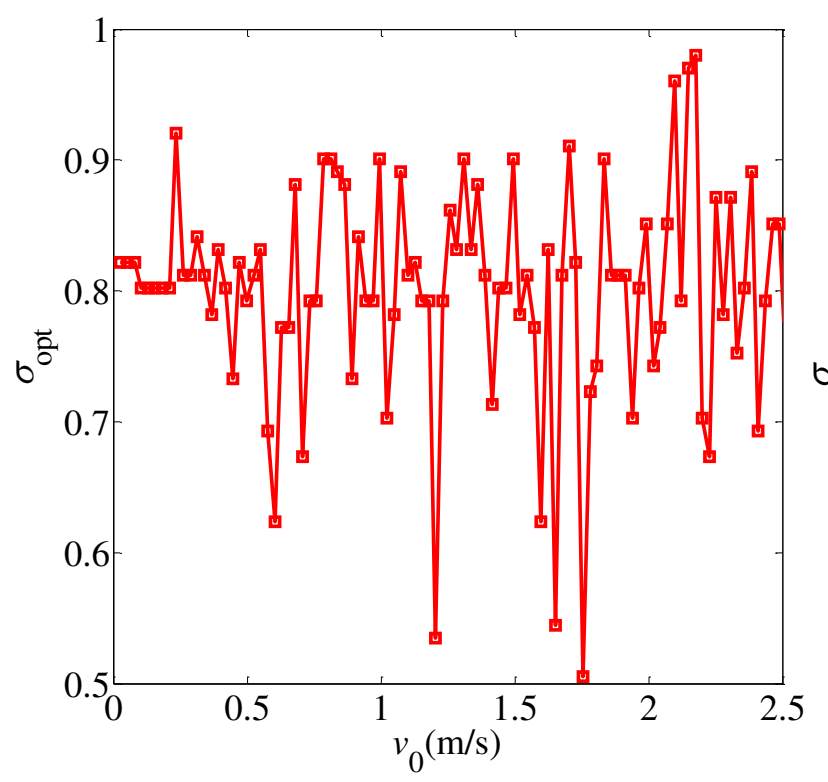

(c)

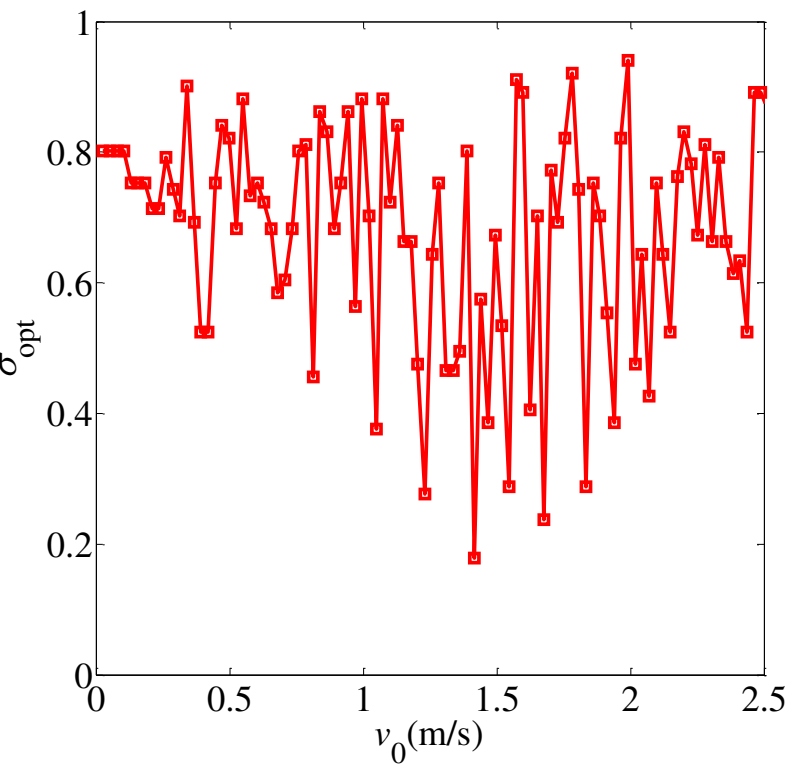

(b)

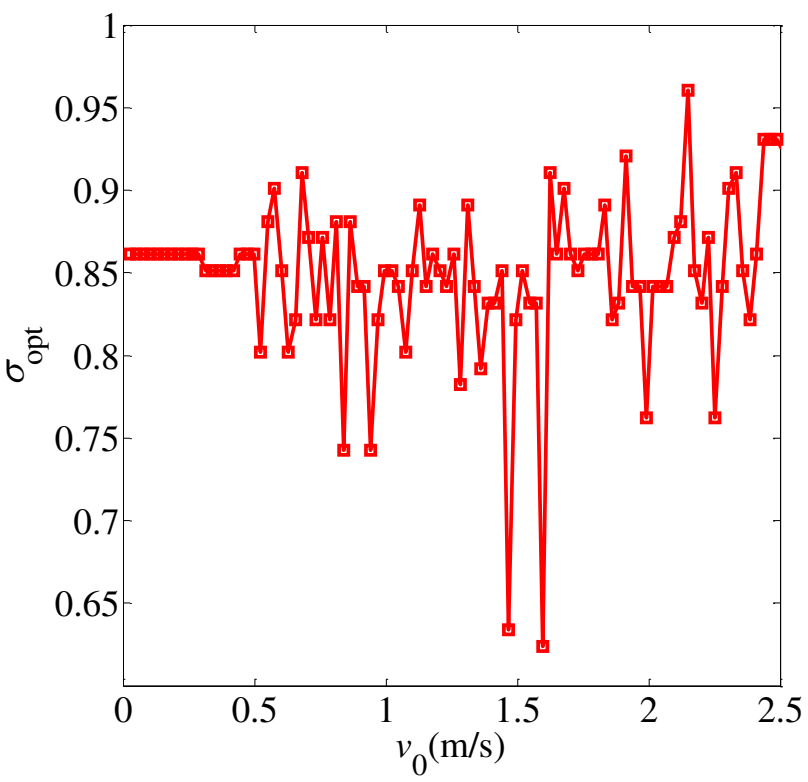

(d)

Figure 21 The optimal length ratio for the minimum residual kinetic energy ratio $\eta_{\mathrm{k}}$ with $\mu N=0.2$. (a) $N=2$; (b) $N=4$; (c) $N=6$; (d) $N=8$

5.3.2 The optimal vibration absorption and energy conversion performance based on optimal number of EC-DVAs

In this section the objective function is defined as the maximum energy conversion efficiency or the minimum residual kinetic energy ratio. The optimization strategy (described in Figure 4 and Figure 5) is to modulate the number of EC-DVAs according to the change of ambient input energy to obtain the desired objective function. The control group is composed of a system with two EC-DVA cells, a system with four EC-DVA cells and a system with eight EC-DVA cells. Hereby, the ratio of total clustered EC-DVAs mass and primary system mass is set to 0.2 . Figure 22 calculates the optimal number of EC-DVAs for the maximum energy conversion efficiency $\eta_{\mathrm{h}}$ with $\mu N=0.2$. From Figure 22 it can be evidently seen that the optimal number of EC-DVAs increases along with the increase in length ratio, and it also found that the optimal number of EC-DVAs is large when the ambient input energy is low and the length ratio is large(greater than 0.9 ). When the length ratio is large the optimal number of EC-DVAs decreases with 
the increase in ambient input energy, as shown in Figure 22.

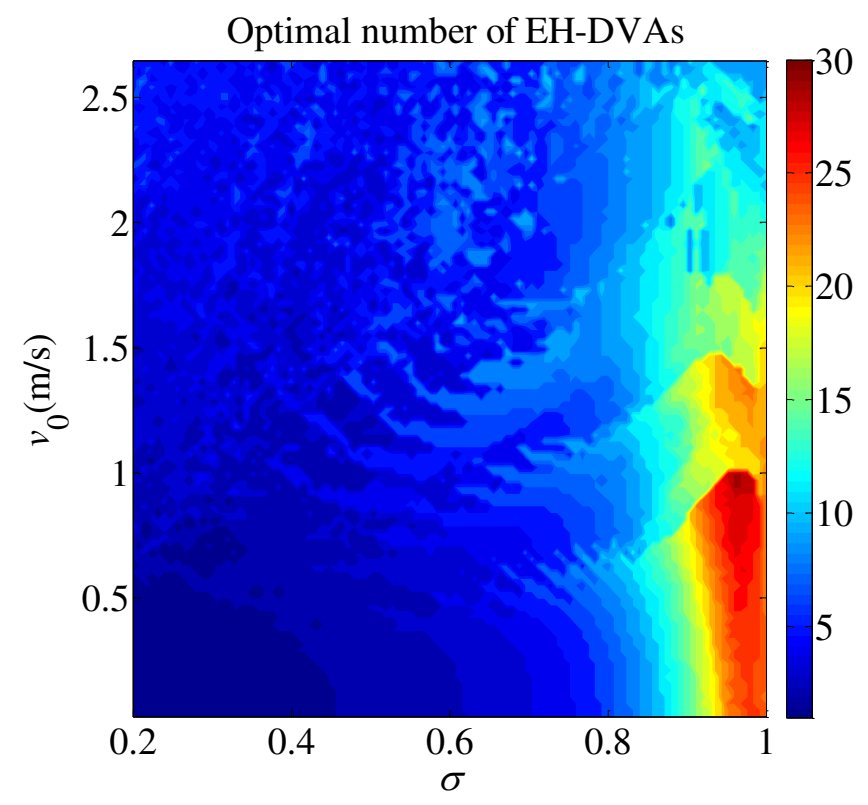

Figure 22 The optimal number of EC-DVAs mapping for the maximum energy conversion efficiency $\eta_{\mathrm{h}}$ with

$$
\mu N=0.2 \text {. }
$$

In order to understand the advantages of this optimization strategy, energy conversion efficiency $\eta_{\mathrm{h}}$ and residual kinetic energy ratio $\eta_{\mathrm{k}}$ resulting from the application of a single impulse to the primary linear system are estimated, respectively. Figure 23 presents color plots of energy conversion efficiency $\eta_{\mathrm{h}}$ resulting from the application of a single impulse to the primary linear system, evaluated at $\tau=60$ as function of impact velocity $v_{0}$ and mass ratio $\mu$ with $\mu N=0.2$. From Figure 23 it can be seen that large number of EC-DVAs is conducive to obtaining desirable energy conversion efficiency when the length ratio of each EC-DVA is large, and small number of EC-DVAs is beneficial to obtaining satisfactory energy conversion efficiency when the length ratio of each EC-DVA is small. Thereby, the number of EC-DVAs can be modulated to obtain the optimal energy conversion performance. Figure 23(d) gives the energy conversion efficiency after this optimization strategy from which it can be easily seen that the energy conversion performance of the system maintains high efficiency and stable when the ambient input energy or the potential energy of clustered EC-DVAs varies.

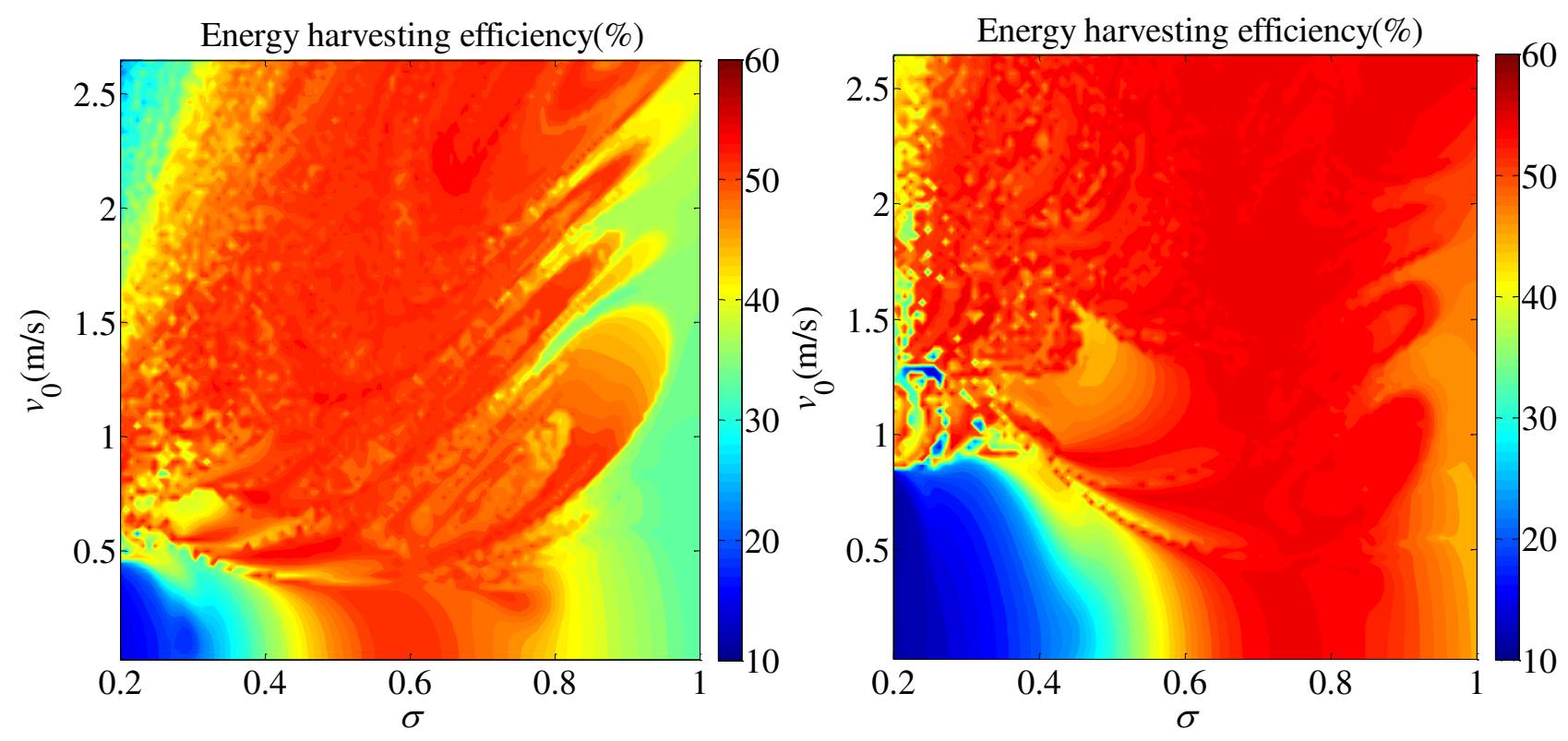


(a)

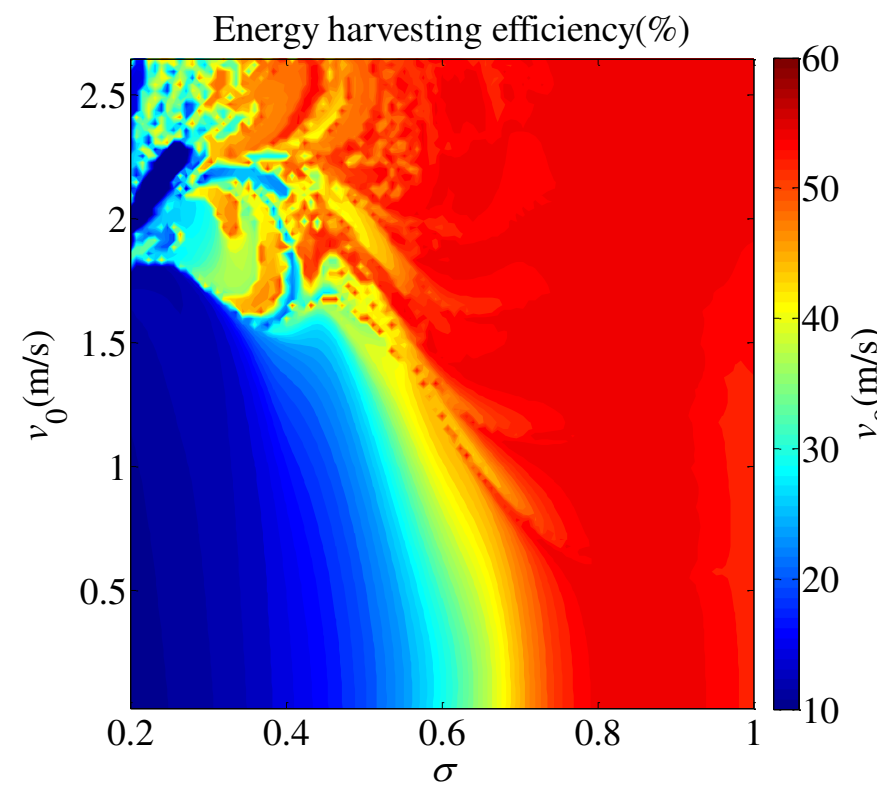

(c) (b)

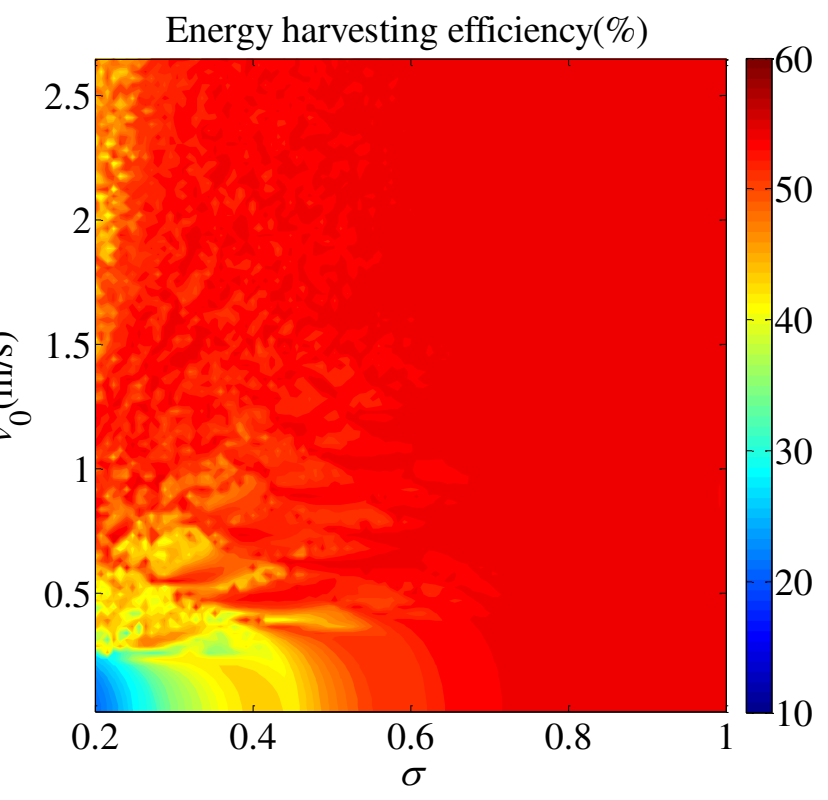

(d)

Figure 23 Color plots of energy conversion efficiency $\eta_{\mathrm{h}}$ resulting from the application of a single impulse to the primary linear system, evaluated at $\tau=60$ as function of impact velocity $v_{0}$ and mass ratio $\mu$ with $\mu N=0.2$. (a) $N=2$;

$$
\text { (b) } N=4 \text {; (c) } N=8 \text {; (d) Optimal }
$$

The alternative way to evaluate the vibration absorption performance is calculating the residual kinetic energy ratio of the primary system after a period of time. Figure 24 shows the optimal number of EC-DVAs for the minimum residual kinetic energy ratio $\eta_{\mathrm{k}}$ with $\mu N=0.2$. The main difference between Figure 22 and Figure 24 is that large optimal number of EC-DVAs is observed in Figure 24 when the length ratio is too small.

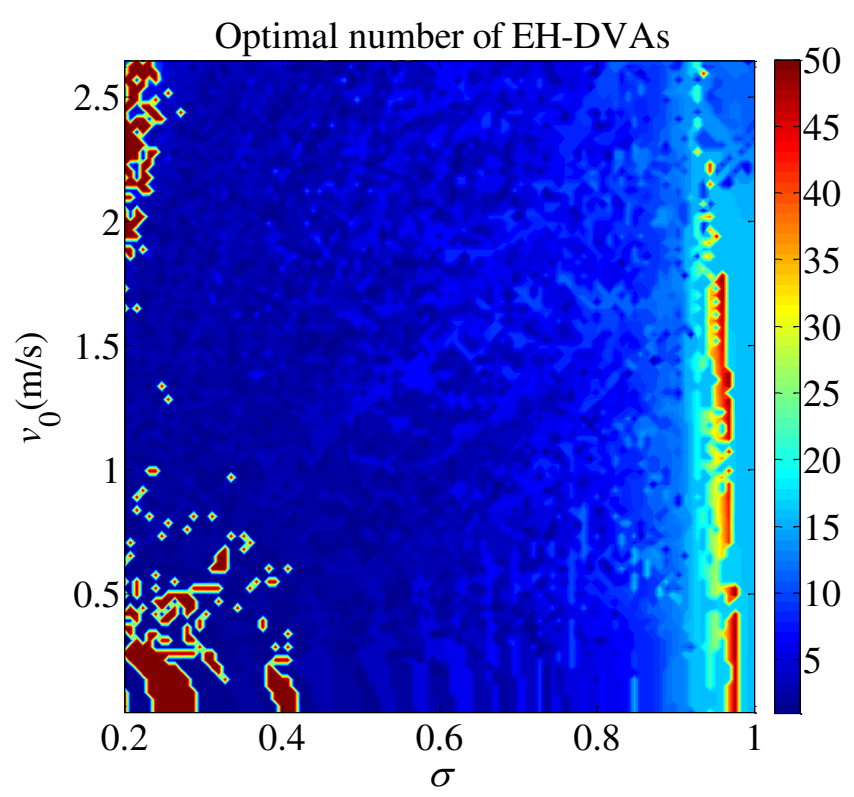

Figure 24 The optimal number of EC-DVAs mapping for the minimum residual kinetic energy ratio $\eta_{\mathrm{k}}$ with

$$
\mu N=0.2 \text {. }
$$

Figure 25 presents color plots of residual kinetic energy ratio $\eta_{\mathrm{k}}$ resulting from the application of a single impulse to the primary linear system, evaluated at $\tau=60$ as function of impact velocity $v_{0}$ and mass ratio $\mu$ with $\mu N=0.2$. From Figure 25 it can be obviously found that the vibration absorption performance of clustered EC-DVAs with too low or 
too large length ratio is barely satisfactory when the number of EC-DVAs is small. The vibration absorption performance of clustered EC-DVAs with large length ratio is improved significantly when the number of EC-DVAs increases, nevertheless, the vibration absorption performance of clustered EC-DVAs with small length ratio is discounted remarkably. Figure 25(d) presents the calculated results of residual kinetic energy ratio after this optimization strategy from which it can be easily seen the high-efficiency vibration absorption performance is sustained and stable when the ambient input energy or the potential energy of clustered EC-DVAs varies. Therefore, this optimization strategy based on optimal number of EC-DVAs is conducive to enhancing vibration suppression of the primary system and improving energy conversion efficiency of clustered EC-DVAs.

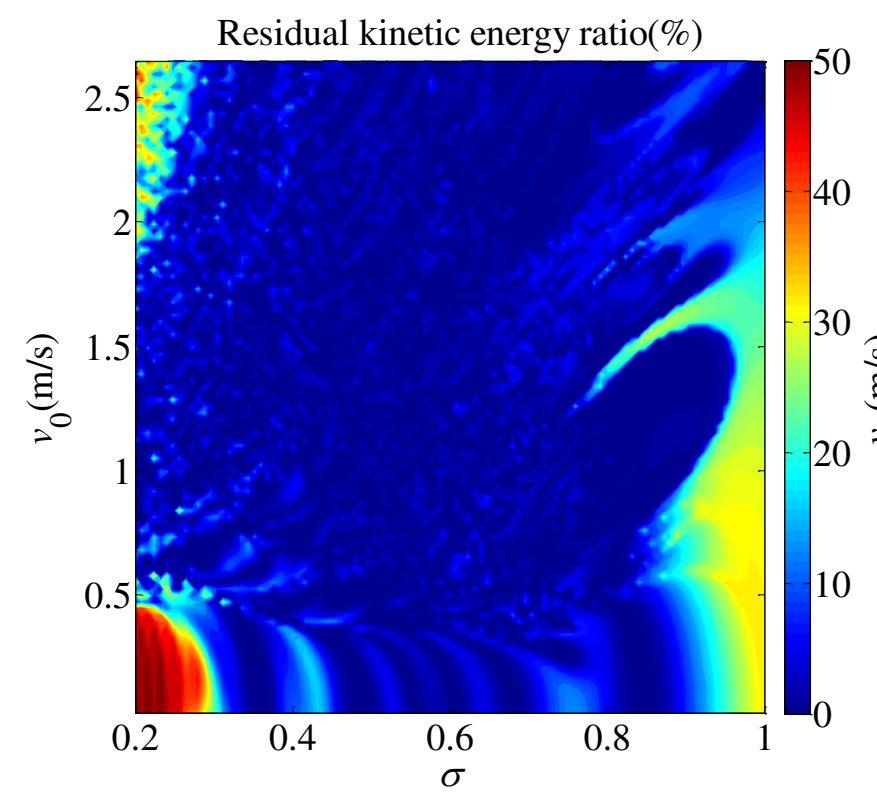

(a)

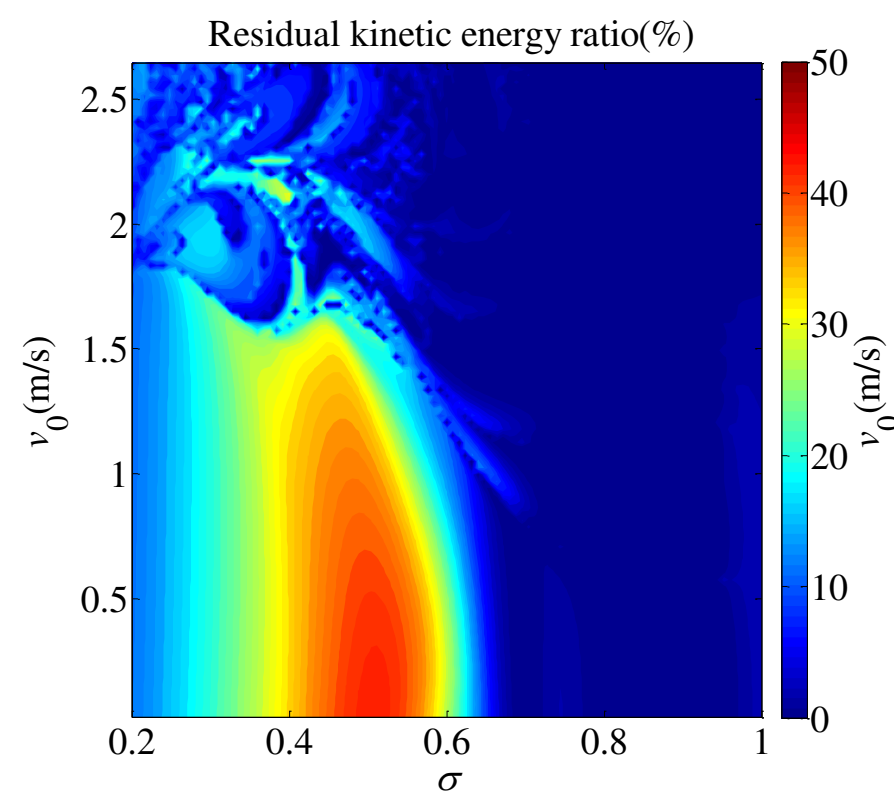

(c)

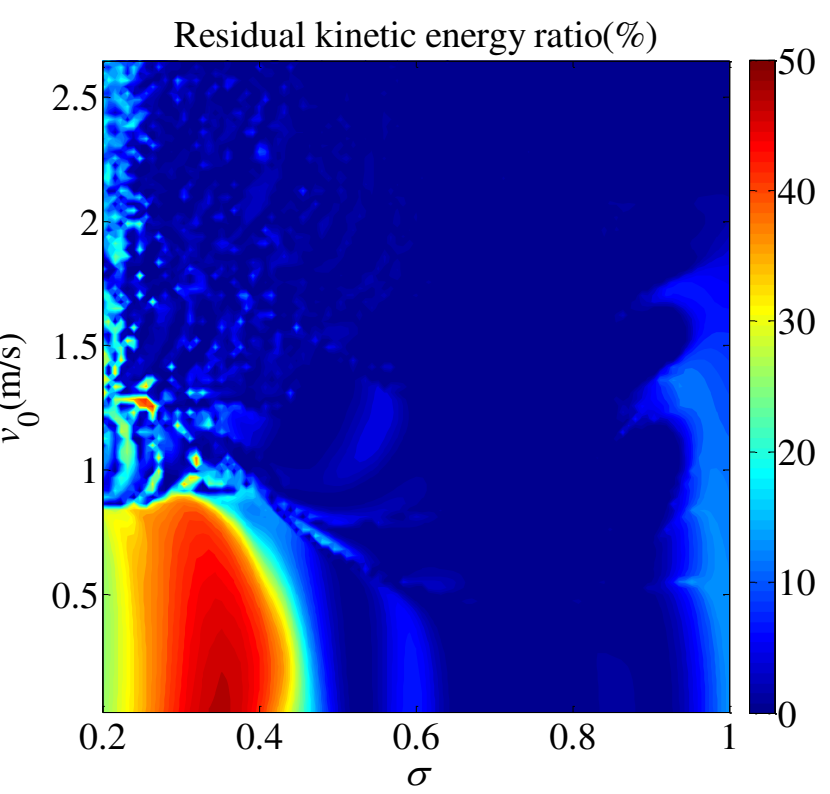

(b)

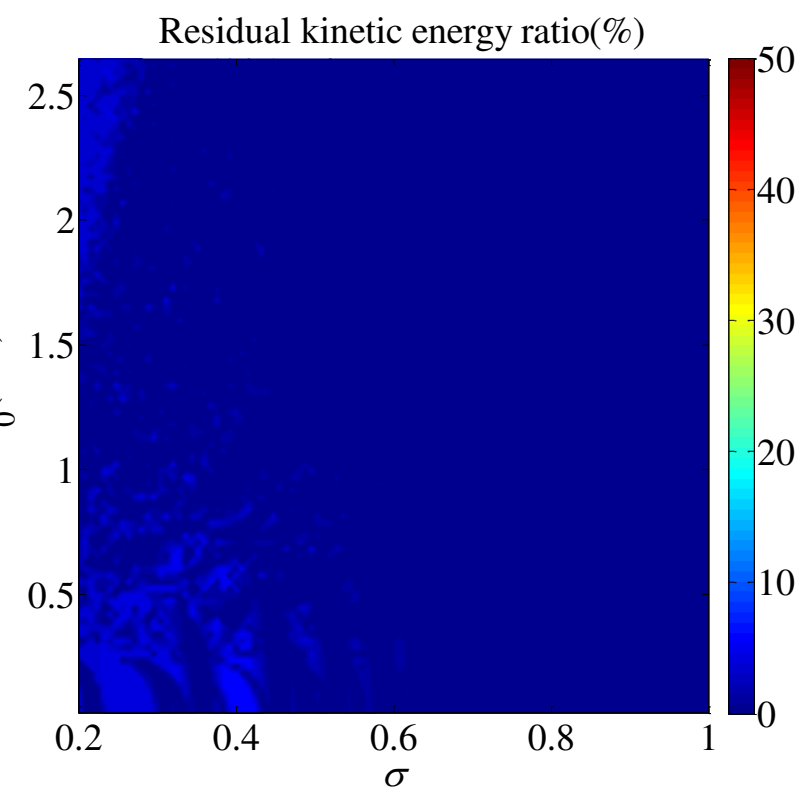

(d)

Figure 25 Color plots of residual kinetic energy ratio $\eta_{\mathrm{k}}$ resulting from the application of a single impulse to the primary linear system, evaluated at $\tau=60$ as function of impact velocity $v_{0}$ and mass ratio $\mu$ with $\mu N=0.2$. (a) $N=2$; (b) $N=4$; (c) $N=8$; (d) Optimal

5.3.3 The optimal vibration absorption and energy conversion performance based on optimal mass ratio 
In this section we investigated the influences of mass ratio on the energy conversion efficiency and vibration migration efficiency, and explored the optimization method based on mass ratio to obtain the most satisfactory vibration absorption and energy conversion performance. The number of EC-DVAs is set to 4 in this section. Hereby, the objective function is also given as the maximum energy conversion efficiency or the minimum residual kinetic energy ratio. The optimization strategy (described in Figure 4 and Figure 5) is to modulate mass ratio according to the variations of ambient input energy to obtain the desired objective function. Figure 26 gives the optimal mass ratio mapping for the maximum energy conversion efficiency, from which it can be found that the optimal mass ratio is large in the conditions of small length ratio and low input energy. When the system has small potential barrier namely large length ratio, the optimal mass ratio is significantly decreased. However, when the system degrades into monostability and the input energy is small scale, the optimal mass ratio is abnormally increased.

Optimal mass ratio

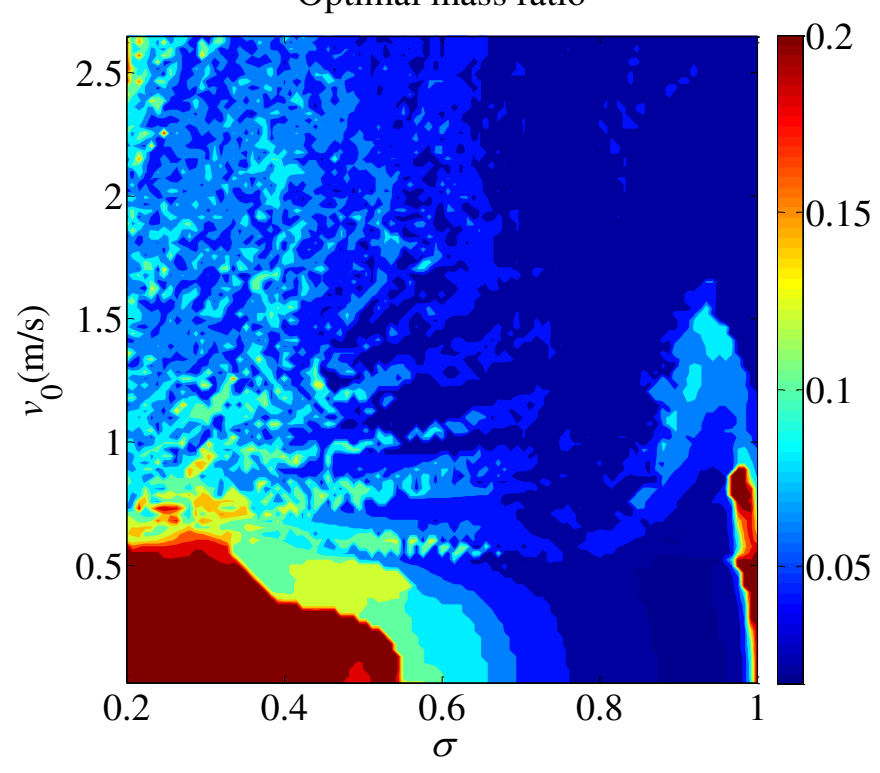

Figure 26 The optimal mass ratio mapping for the maximum energy conversion efficiency $\eta_{\mathrm{h}}$

Figure 27 presents color plots of energy conversion efficiency $\eta_{\mathrm{h}}$ resulting from the application of a single impulse to the primary linear system, evaluated at $\tau=60$ as function of impact velocity $v_{0}$ and length ratio $\sigma$ with $N=4$. From Figure 27 it can be easily seen that the increase in mass ratio induces remarkable enhancements in energy conversion efficiency for those systems with large potential barrier, and also found that energy conversion efficiency of clustered EC-DVAs is satisfactory when the mass ratio is increased to 0.1. From Figure 27(d) it can be seen that the energy conversion performance can be obviously improved by means of tuning the mass ratio to its optimum compared with the control group. 


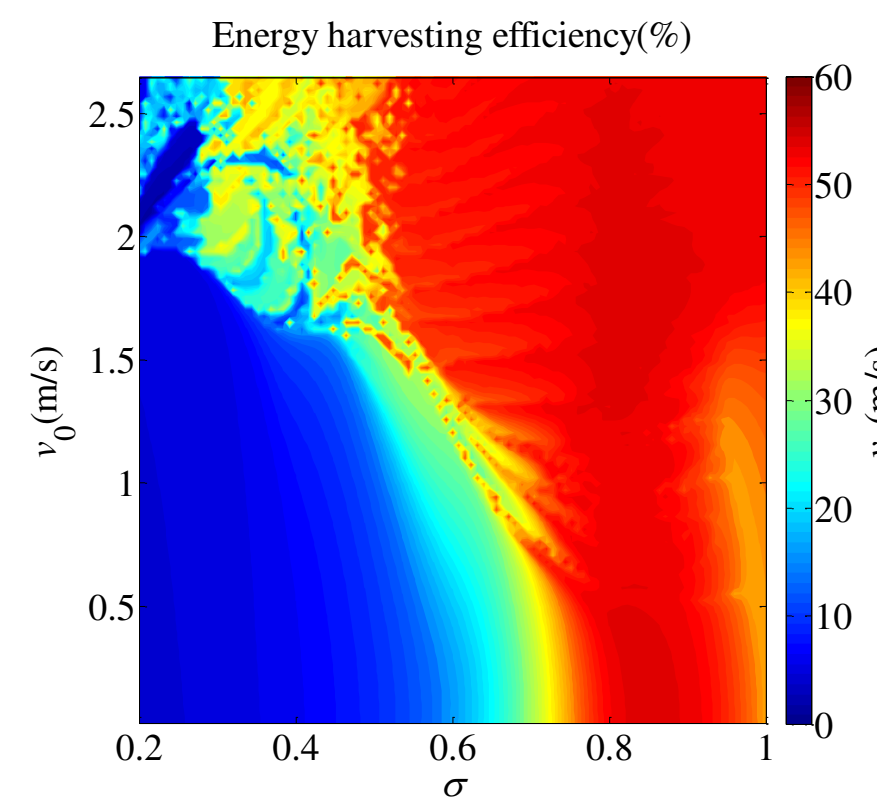

(a)

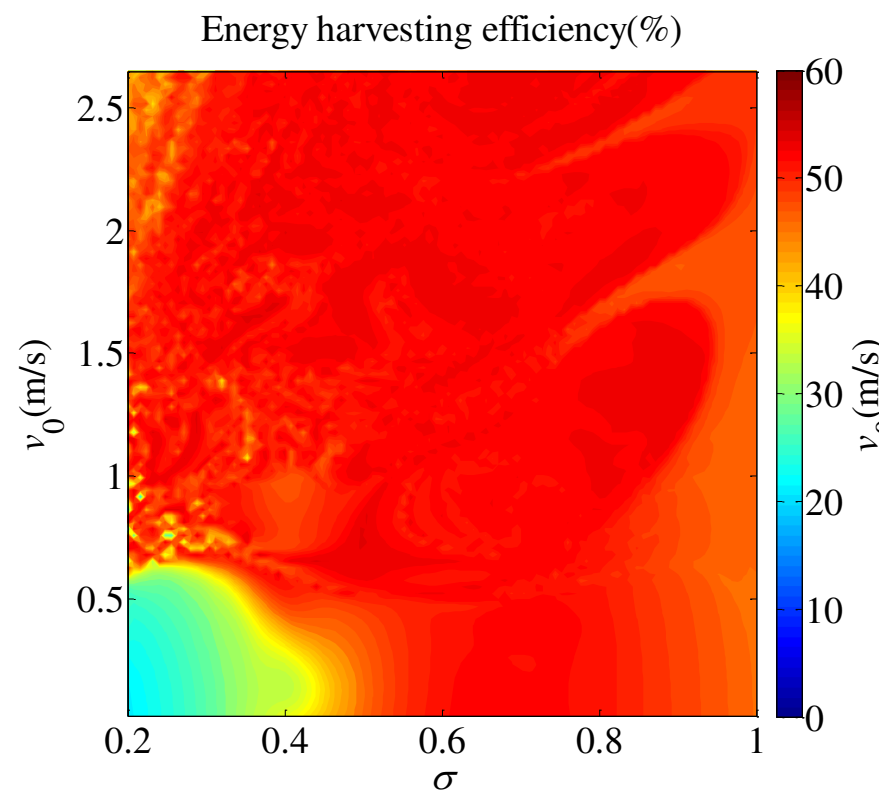

(c)

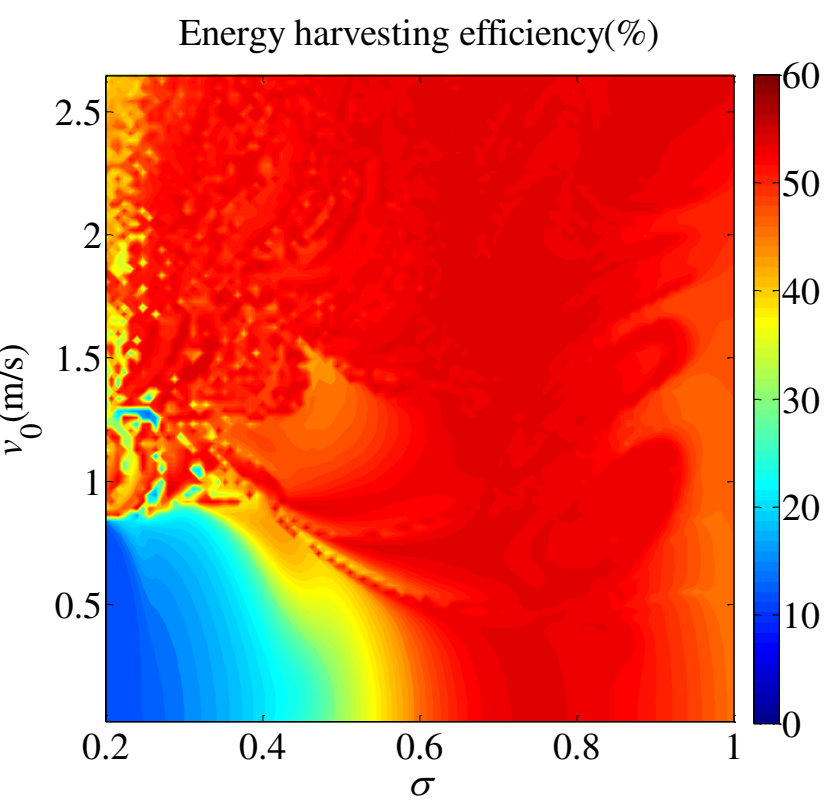

(b)

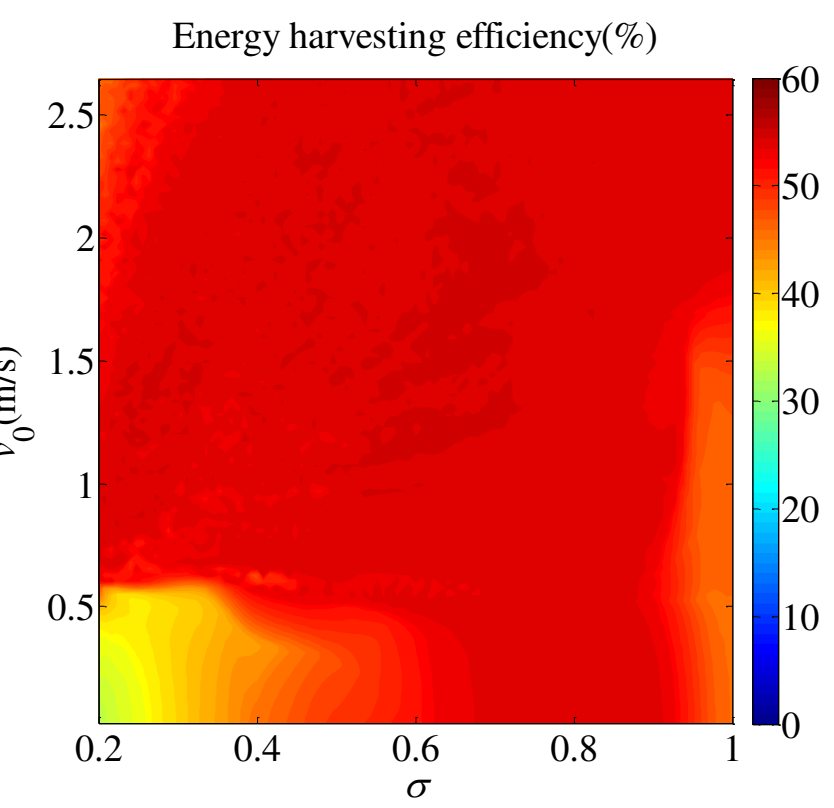

(d)

Figure 27 Color plots of energy conversion efficiency $\eta_{\mathrm{h}}$ resulting from the application of a single impulse to the primary linear system, evaluated at $\tau=60$ as function of impact velocity $v_{0}$ and length ratio $\sigma$ with $N=4$. (a) $\mu=0.02$;

(b) $\mu=0.05$; (c) $\mu=0.1$; (d) Optimal

Figure 28 shows the optimal mass ratio mapping for the minimum residual kinetic energy ratio, from which it can be found that the optimal mass ratio is in scattered distribution. When the length ratio is close to land the impact speed is under $1.4 \mathrm{~m} / \mathrm{s}$, the optimal mass ratio increases greatly. Figure 29 presents color plots of residual kinetic energy ratio $\eta_{\mathrm{k}}$ resulting from the application of a single impulse to the primary linear system, evaluated at $\tau=60$ as function of impact velocity $v_{0}$ and length ratio $\sigma$ with $N=4$. From Figure 29 it can be evidently seen that the residual kinetic energy ratio decreases remarkably with the increase in mass ratio, and also found the residual kinetic energy ratio is extensively reduced when the mass ratio is set to 0.1 . Figure 29 (d) gives the optimum results of vibration absorption and energy transfer, from which it can be seen uniformly blue regions that denote low residual kinetic energy in the primary system. Therefore, the optimization results based on both the maximum energy conversion 
efficiency and minimum residual kinetic energy ratio objectives are similar and in good agreements.

Optimal mass ratio

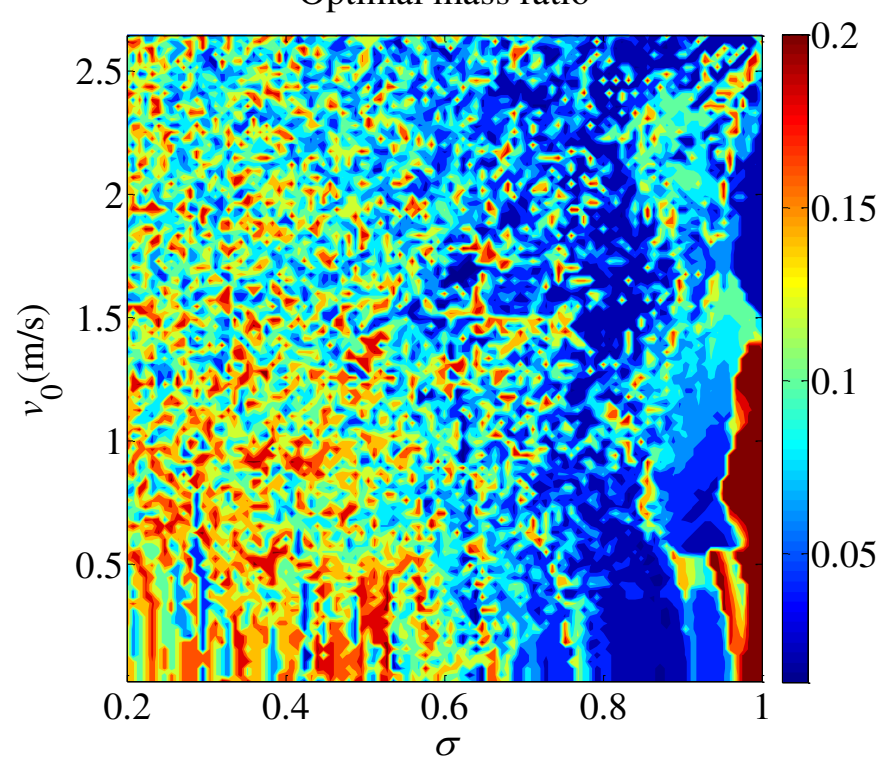

Figure 28 The optimal mass ratio mapping for the minimum residual kinetic energy ratio $\eta_{\mathrm{k}}$

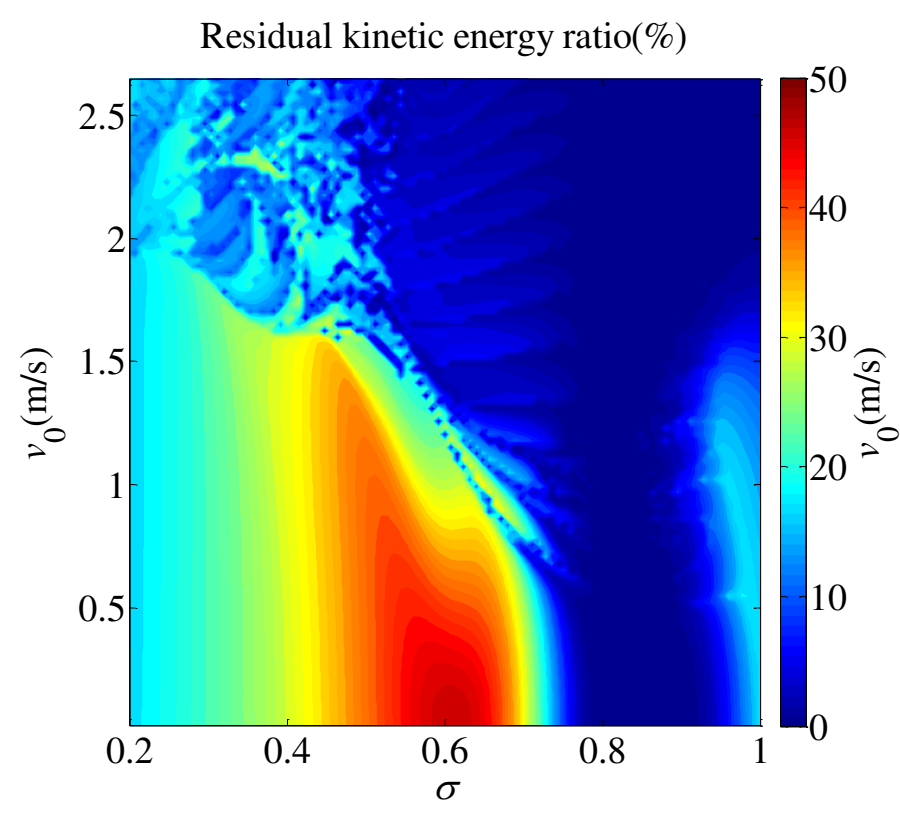

(a)
Residual kinetic energy ratio(\%)

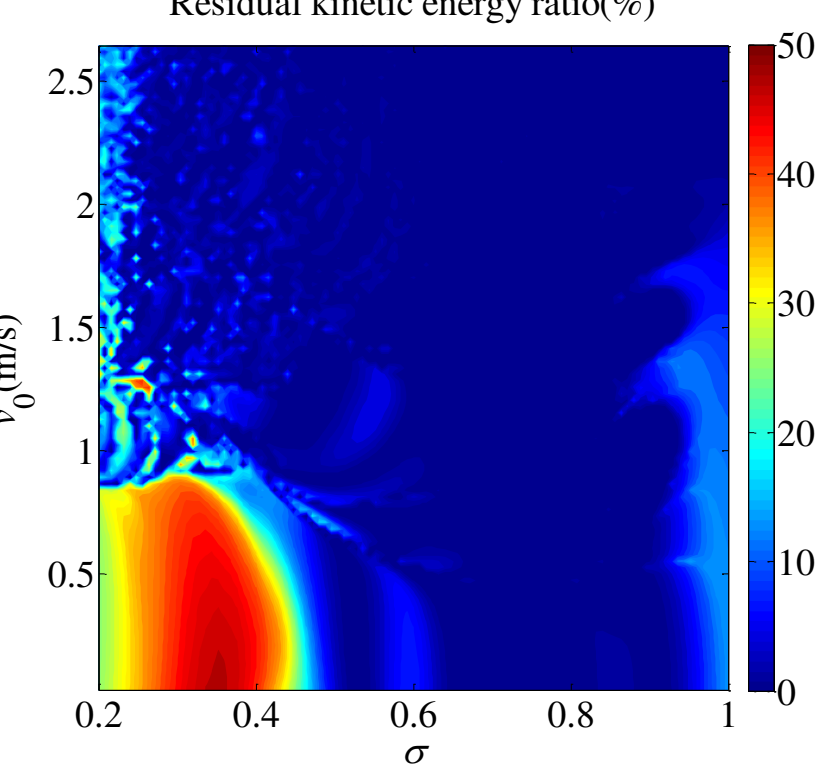

(b) 


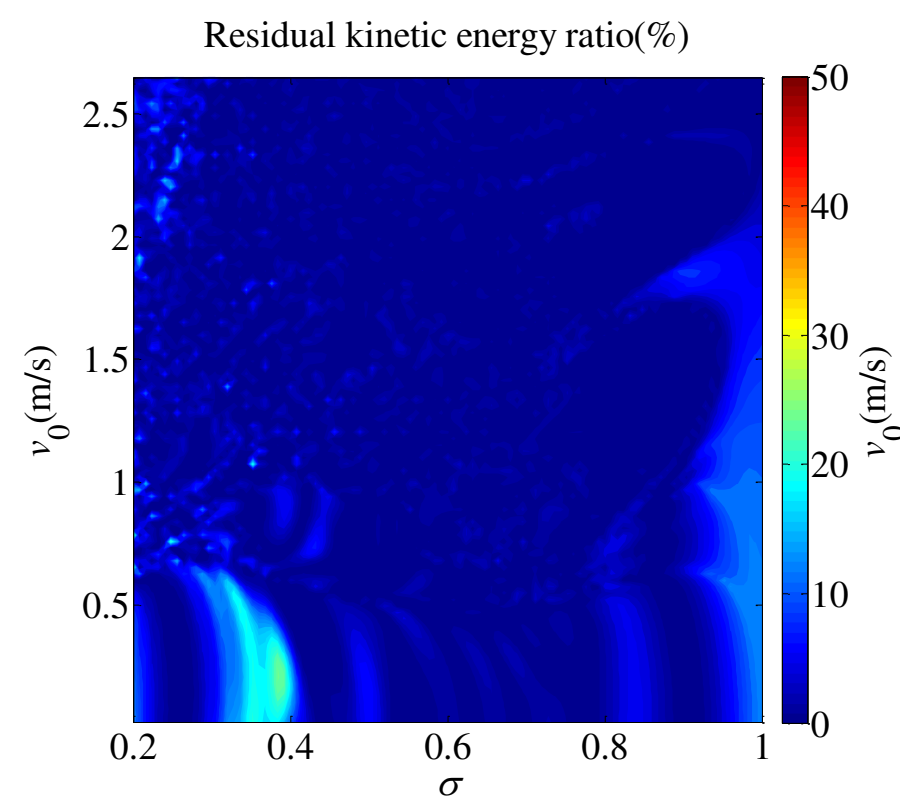

(c)

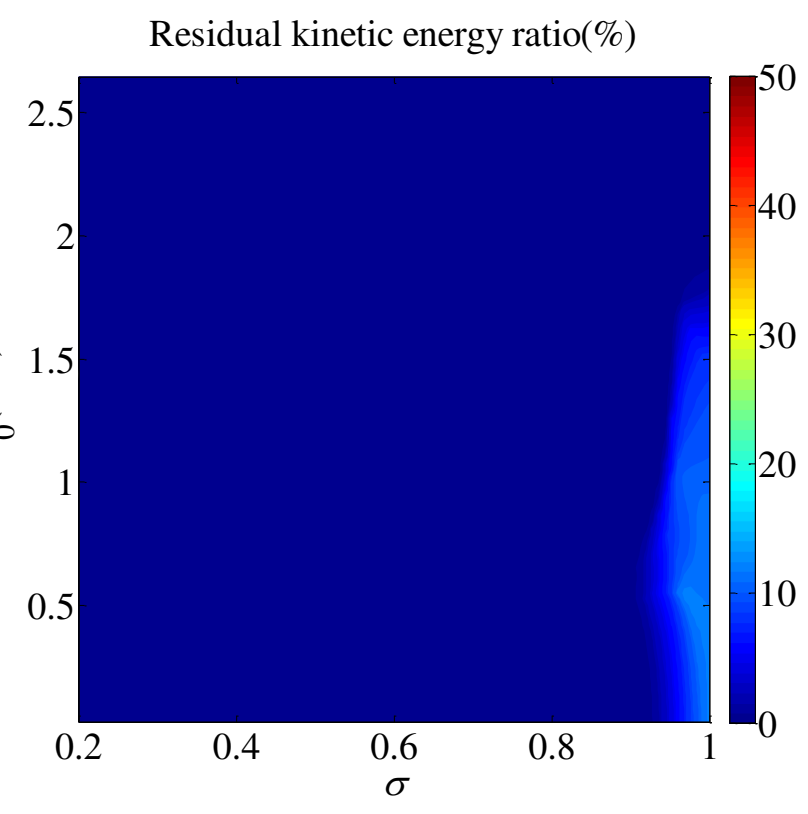

(d)

Figure 29 Color plots of residual kinetic energy ratio $\eta_{\mathrm{k}}$ resulting from the application of a single impulse to the primary linear system, evaluated at $\tau=60$ as function of impact velocity $v_{0}$ and length ratio $\sigma$ with $N=4$. (a) $\mu=0.02$;

(b) $\mu=0.05$; (c) $\mu=0.1$; (d) Optimal

\section{Concluding remarks}

This paper proposes a flexible energy conversion inspired vibration control methodology by introducing a tunable bi-stable structure. The transient TET performance and nonlinear motion behaviors of clustered EC-DVAs are studied firstly, and the influences of length ratio and EC-DVA number on energy conversion and vibration suppression are analyzed. Two optimization strategies, namely adaptive modulation of the bi-stable potential energy and adaptive management of tunable clustered EC-DVAs number are investigated and compared with the control group, respectively. The evaluation criterions composed of the maximum energy conversion efficiency and the minimum residual kinetic energy are considered and studied, respectively. The energy conversion efficiency and residual kinetic energy after dynamic length ratio optimization is independent on ambient input energy and the number of EC-DVAs, however, the variation range of optimal length ratio decreases with the increase in number of EC-DVAs. The energy conversion efficiency and vibration absorption performance based on optimal number of ECDVAs maintain high efficiency and stable when the ambient input energy or the potential energy of clustered ECDVAs varies. Large mass ratio causes great energy conversion efficiency and small residual kinetic energy ratio when the system's potential barrier is too large and the ambient input energy is small. The optimization results of two optimization strategies based on the mass ratio are satisfactory. Therefore, the two optimization strategies are potential to becoming the embedded control criterion for changing the bi-stable potential energy, tuning the mass ratio and number of EC-DVAs to implement satisfactory vibration absorption and energy conversion performance.

\section{Acknowledgement}

The work is supported by the National Key R\&D Program of China (2017YFF0108000) and National Natural Science Foundation of China (5177349), for which the authors are most grateful.

\section{Declarations}

No conflict of interest exists in the submission of this manuscript, and manuscript is approved by all authors for publication. I would like to declare on behalf of my co-authors that the work described was original research that has not been published previously, and not under consideration for publication elsewhere, in whole or in part. 


\section{Data Availability Statement}

All relevant supporting data and available information in this manuscript can be acquired through contacting the Corresponding author.

\section{References}

[1] K. T. Tse, K. C. S. Kwok, Y. Tamura. Performance and cost evaluation of a smart tuned mass damper for suppressing wind-induced lateral-torsional motion of tall structures[J]. Journal of Structural Engineering, 138(4)(2012): 514-525.

[2] N. D. Tiwari, A. Gogoi, B. Hazra, Q. Wang. A shape memory alloy-tuned mass damper inerter system for passive control of linked-SDOF structural systems under seismic excitation[J]. Journal of Sound and Vibration, 494(2021): 115893.

[3] Z. Lu, B. Huang, Q. Zhang, X. Lu. Experimental and analytical study on vibration control effects of eddy-current tuned mass dampers under seismic excitations[J]. Journal of Sound and Vibration, 421(2018): 153-165.

[4] J. Wang, L. Geng, L. Ding, H. Zhu, D. Yurchenko. The state-of-the-art review on energy conversion from flowinduced vibrations[J]. Applied Energy, 267(2020): 114902.

[5] B. Qin, M. M. Alam, C. Ji, Y. Liu, S. Xu. Flow-induced vibrations of two cylinders of different natural frequencies[J]. Ocean Engineering, 155(2018): 189-200.

[6] J. Zhou, M. Xu, Z. Yang, Y. Gu. Suppression of panel flutter response in supersonic airflow using a nonlinear vibration absorber[J]. International Journal of Non-Linear Mechanics, 133(2021): 103714.

[7] A. Heng, S. Zhang, A. C. C. Tan, J. Mathew. Rotating machinery prognostics: State of the art, challenges and opportunities[J]. Mechanical systems and signal processing, 23(3)(2009): 724-739.

[8] Y. Lei, J. Lin, Z. He, M. J. Zuo. A review on empirical mode decomposition in fault diagnosis of rotating machinery[J]. Mechanical systems and signal processing, 35(1-2)(2013): 108-126.

[9] K. V. Nguyen, H. T. Tran. Multi-cracks detection of a beam-like structure based on the on-vehicle vibration signal and wavelet analysis[J]. Journal of Sound and Vibration, 329(21)(2010): 4455-4465.

[10] S. D. Nguyen, B. D. Lam, S. B. Choi. Smart dampers-based vibration control-Part 2: Fractional-order sliding control for vehicle suspension system[J]. Mechanical Systems and Signal Processing, 148(2021): 107145.

[11] M. F. Liu, T. P. Chang, D. Y. Zeng. The interactive vibration behavior in a suspension bridge system under moving vehicle loads and vertical seismic excitations[J]. Applied Mathematical Modelling, 35(1)(2011): 398-411.

[12] Y. Cao, D. Cao, G. He, L. Liu. Thermal alternation induced vibration analysis of spacecraft with lateral solar arrays in orbit[J]. Applied Mathematical Modelling, 86(2020): 166-184.

[13] H. Peng, Y. Liu, H. Chen, J. Shen. Shakedown analysis of engineering structures under multiple variable mechanical and thermal loads using the stress compensation method[J]. International Journal of Mechanical Sciences, 140(2018): 361-375.

[14] S. Y. Kim, C. H. Lee. Optimum design of linear multiple tuned mass dampers subjected to white-noise base acceleration considering practical configurations[J]. Engineering Structures, 171(2018): 516-528.

[15] Zuo L. Effective and robust vibration control using series multiple tuned-mass dampers[J]. Journal of Vibration and Acoustics, 131(3)(2009): 031003.

[16] S. Chiacchiari, F. Romeo, D. M. McFarland, L. A. Bergman, A. F. Vakakis. Vibration energy conversion from impulsive excitations via a bistable nonlinear attachment[J]. International Journal of Non-Linear Mechanics, 94(2017): 84-97.

[17] S. Chiacchiari, F. Romeo, McFarland D M, et al. Vibration energy conversion from impulsive excitations via a bistable nonlinear attachment—Experimental study[J]. Mechanical Systems and Signal Processing, 125(2019): 185-201.

[18] X. Huang, B. Yang. Investigation on the energy trapping and conversion performances of a multi-stable vibration 
absorber[J]. Mechanical Systems and Signal Processing, 160(2021): 107938.

[19] H. T. Nguyen, D. Genov, H. Bardaweel. Mono-stable and bi-stable magnetic spring based vibration energy conversion systems subject to harmonic excitation: Dynamic modeling and experimental verification[J]. Mechanical Systems and Signal Processing, 134(2019): 106361.

[20] Y. Hua, W. Wong, L. Cheng. Optimal design of a beam-based dynamic vibration absorber using fixed-points theory[J]. Journal of Sound and Vibration, 421(2018): 111-131.

[21] Y. Shen, Z. Xing, S. Yang, J. Sun. Parameters optimization for a novel dynamic vibration absorber[J]. Mechanical Systems and Signal Processing, 133(2019): 106282.

[22] M. X. He, X. Lyu, Y. Zhai, Y. Tang, T. Yang, Q. Ding. Multi-objective optimal design of periodically stiffened panels for vibration control using data-driven optimization method[J]. Mechanical Systems and Signal Processing, 160(2021):107872. 